\title{
On squares of representations of compact Lie algebras
}

\author{
Robert Zeiera) \\ Department Chemie, Technische Universität München, \\ Lichtenbergstrasse 4, 85747 Garching, Germany \\ Zoltán Zimborás) \\ Department of Computer Science, University College London, \\ Gower St, London WC1E 6BT, UK
}

(Dated: 24 August 2015)

\begin{abstract}
We study how tensor products of representations decompose when restricted from a compact Lie algebra to one of its subalgebras. In particular, we are interested in tensor squares which are tensor products of a representation with itself. We show in a classification-free manner that the sum of multiplicities and the sum of squares of multiplicities in the corresponding decomposition of a tensor square into irreducible representations has to strictly grow when restricted from a compact semisimple Lie algebra to a proper subalgebra. For this purpose, relevant details on tensor products of representations are compiled from the literature. Since the sum of squares of multiplicities is equal to the dimension of the commutant of the tensor-square representation, it can be determined by linear-algebra computations in a scenario where an a priori unknown Lie algebra is given by a set of generators which might not be a linear basis. Hence, our results offer a test to decide if a subalgebra of a compact semisimple Lie algebra is a proper one without calculating the relevant Lie closures, which can be naturally applied in the field of controlled quantum systems.
\end{abstract}

\section{INTRODUCTION}

The work of Dynkin ${ }^{1-3}$ is a treasure trove of useful information on representations of Lie algebras. In particular, Ref. 1 enumerates all representations whose alternating square is irreducible (see Table \below). This classification triggered in Ref. 4 a study of tensor squares $\phi \otimes \phi$ which are defined for a representation $\phi$ of a Lie algebra $\mathfrak{g}$ as the representation $(\phi \otimes \phi)(g):=\phi(g) \otimes \mathbb{1}_{\operatorname{dim}(\phi)}+\mathbb{1}_{\operatorname{dim}(\phi)} \otimes \phi(g)$ with $g \in \mathfrak{g}$. The tensor square $\phi \otimes \phi$ of the standard (i.e., defining) representation of the Lie algebra $\mathfrak{s u}(\ell+1)$ corresponding to the special unitary group has the property that the dimension of its commutant $\mathfrak{c o m}[\phi \otimes \phi]$ has to grow when restricted to a proper subalgebra $\mathfrak{h}$, i.e., $\operatorname{dim}\left(\mathfrak{c o m}\left[\left.(\phi \otimes \phi)\right|_{\mathfrak{h}}\right]\right)>\operatorname{dim}(\mathfrak{c o m}[\phi \otimes \phi])=2$. Here, $\mathfrak{c o m}[\psi]$ denotes the commutant of a representation $\psi$ of a Lie algebra $\mathfrak{g}$ and consists of all complex matrices commuting with all $\psi(g)$ for $g \in \mathfrak{g}$. This discussion can be summarized as follows.

Theorem A (see Thm. 21 of Ref. 4). Given a subalgebra $\mathfrak{h}$ of $\mathfrak{s u}(\ell+1)$ with $\ell \geq 1$ and the standard representation $\phi$ of $\mathfrak{s u}(\ell+1)$, then $\mathfrak{h}=\mathfrak{s u}(\ell+1)$ iff $\operatorname{dim}\left(\mathfrak{c o m}\left[\left.(\phi \otimes \phi)\right|_{\mathfrak{h}}\right]\right)=2$.

The power of Theorem $\mathrm{A}$ arises from the fact that its condition can be tested using only a set of generators for the Lie algebra $\mathfrak{h}$, as those generators are sufficient to compute the commutant $\operatorname{com}\left[\left.(\phi \otimes \phi)\right|_{\mathfrak{h}}\right]$ of the tensor square. This led to control-theoretic applications in Ref. 4 where a controlled Schrödinger equation provides certain initial directions (i.e., Lie-algebra generators) in which a quantum system can be steered. In general, these directions do not linearly span but only generate the a priori unknown Lie algebra of all achievable directions, e.g., the threedimensional, infinitesimal rotations around the $x$ - and $y$-axes generate an additional infinitesimal rotation around the $z$-axis via the Lie commutator. In this context, one wants to decide effectively if the full Lie algebra $\mathfrak{s u}(\ell+1)$ is generated without using the standard technique of computing the cumbersome Lie closure for a given set of generators. With the help Theorem $\mathrm{A}$, the question if a subalgebra of $\mathfrak{s u}(\ell+1)$ is a proper one can now be completely reduced to linear-algebra computations. The proof given in Ref. 4 borrows heavily from the classification of alternating squares in Dynkin's work ${ }^{1}$ and treats all cases individually. The motivation of the current work is to better understand the basic principles on which Theorem $\mathrm{A}$ relies. In doing so, we identify the following generalization of Theorem $\mathrm{A}$ where $\mathfrak{s u}(\ell+1)$ is substituted by an arbitrary compact, semisimple Lie algebra $\mathfrak{g}$ and the standard representation of $\mathfrak{s u}(\ell+1)$ is replaced with an arbitrary finite-dimensional, faithful representation of $\mathfrak{g}$.

Theorem B. Given a subalgebra $\mathfrak{h}$ of a compact semisimple Lie algebra $\mathfrak{g}$ and a finite-dimensional, faithful representation $\phi$ of $\mathfrak{g}$, then $\mathfrak{h}=\mathfrak{g}$ iff $\operatorname{dim}\left(\mathfrak{c o m}\left[\left.(\phi \otimes \phi)\right|_{\mathfrak{h}}\right]\right)=\operatorname{dim}(\mathfrak{c o m}[\phi \otimes \phi])$.

\footnotetext{
a) Electronic mail: robert.zeier@ch.tum.de

b) Electronic mail: zimboras@gmail.com
} 
Moreover, the proof of Theorem B does not rely on information from classifications and highlights general properties of restricted representations for compact, semisimple Lie algebras and beyond. Limitations on potential generalizations to arbitrary compact Lie algebras will be discussed in Section IV Theorems A and B can be naturally transferred to connected, compact semisimple Lie groups, as their representations induce always a semisimple representation of the corresponding compact semisimple Lie algebra. Let us note, however, that the above theorems are not trivial consequences of the representation theory of general compact groups; in particular, they do not hold for finite groups. We provide a counter-example for non-connected compact groups (recall that finite groups are formally non-connected, zero-dimensional Lie groups): Consider the set $\mathcal{M}=\left\{ \pm\left(\begin{array}{ll}1 & 0 \\ 0 & 1\end{array}\right), \pm\left(\begin{array}{cc}i & 0 \\ 0 & -i\end{array}\right), \pm\left(\begin{array}{cc}0 & -1 \\ 1 & 0\end{array}\right), \pm\left(\begin{array}{cc}0 & i \\ i & 0\end{array}\right), \frac{1}{2}\left( \pm\left(\begin{array}{cc}1 & 0 \\ 0 & 1\end{array}\right) \pm\left(\begin{array}{cc}i & 0 \\ 0 & -i\end{array}\right) \pm\left(\begin{array}{cc}0 & -1 \\ 1 & 0\end{array}\right) \pm\left(\begin{array}{ll}0 & i \\ i & 0\end{array}\right)\right)\right\}$ of 24 matrices. One can easily check that the matrices of $\mathcal{M}$ form a group $H$. As all generators in $\mathcal{M}$ are contained in $\mathrm{SU}(2)$ (given in its standard representation), $H$ is a proper subgroup of $\mathrm{SU}(2)$. Moreover, $H$ is given here in a particular unitary representation, is a double cover of the tetrahedral group, and is isomorphic to the special linear group $\operatorname{SL}\left(2, \mathbb{F}_{3}\right)$ of $2 \times 2$-matrices with entries from the finite field $\mathbb{F}_{3}$ and with determinant one. Denoting the standard representation of SU(2) by $\phi$, the described representation of $H$ will be naturally identified as $\left.\phi\right|_{H}$. Let us consider the tensor squares $\left.(\phi \otimes \phi)\right|_{H}$ and $\phi \otimes \phi$ which are defined as $(\phi \otimes \psi)(X):=\phi(X) \otimes \psi(X)$ for elements $X$ of a group $G$. One obtains that $\operatorname{dim}\left(\mathfrak{c o m}\left[\left.(\phi \otimes \phi)\right|_{H}\right]\right)=2=\operatorname{dim}(\mathfrak{c o m}[(\phi \otimes \phi)])$ and shows that Theorems $\mathrm{A}$ and B cannot be generalized to general compact groups which might not be connected. Let us also note that finite subgroups $H$ of $\mathrm{SU}(d)$ with the property that $\operatorname{dim}\left(\mathfrak{c o m}\left[\left.(\phi \otimes \phi)\right|_{H}\right]\right)=\operatorname{dim}(\mathfrak{c o m}[(\phi \otimes \phi)])$ are known as group designs (which are particular types of unitary 2-designs) and have also been studied in the context of quantum information theory. $\underline{\underline{5} .6}$

It is not unusual that limiting compact groups to connected ones leads to significant modifications from a representation-theoretic point of view. For example, it is well-known that there exist non-isomorphic compact groups with isomorphic representation rings. 7 On the other hand, two connected compact groups can only have isomorphic representation rings if the corresponding groups are isomorphic $8-10$ In the case of a connected semisimple (complex) Lie group, it is even enough to determine the so-called dimension datum of a finite-dimensional, faithful representation $\phi$ in order to fix its Lie algebra. ${ }^{11-13}$ The dimension datum corresponds roughly to knowing all dimensions for representations occurring in any tensor power of $\phi$. In this context, it is surprising that the conditions in Theorems $\mathrm{A}$ and $\mathrm{B}$ rely only on the tensor square (but admittedly for a weaker conclusion). Finally, Coquereaux and Zuber $^{14}$ proved properties for the sum of multiplicities in the decomposition of a tensor product of two irreducible representations of a simple Lie algebra (see Appendix (D), which also cannot be generalized to the non-connected group case.

We will assume that the reader has some familiarity with Lie algebras and representations, which are both considered to be finite-dimensional throughout this work. All Lie algebras are defined over the real or complex field, and all representations are matrix representations with complex matrix entries. We will use the words irreducible and simple (as well as completely reducible and semisimple) as interchangeable names for properties of representations; irreducibility is always considered with respect to the complex numbers. For better accessibility, important facts and notations are recalled in Appendix A. Our presentation will focus on compact Lie algebras, although the parallel language of complex reductive Lie algebras would be also suitable to state our results (cf. Appendix A); and we will switch between them when necessary without further comment.

The article is organized as follows: We start in Section II by summarizing a classification of representations with irreducible alternating and symmetric squares; the corresponding details are given in Appendices B and C. The main classification-free results leading to Theorem B are presented in Section III. We close by discussing generalizations to general compact Lie algebras as well as lower bounds on the gap between the dimensions of commutants of tensor square representations. Parts of the discussion are relegated to appendices in order to streamline the presentation.

\section{ALTERNATING, SYMMETRIC, AND TENSOR SQUARES}

In this section, we summarize results classifying representations whose alternating and symmetric tensor squares are simple (i.e., irreducible). Streamlined proofs of these classifications which apply techniques developed by Dynkin (see Appendix B and Ref. 1) are relegated to Appendix C. The classification results allow us to prove techniques for distinguishing $\mathfrak{s o}(k), \mathfrak{s p}(\ell)$, or $\mathfrak{s u}(\ell+1)$ from its subalgebras (cf. Refs. 4 and 15), but we also provide simplified, classification-independent proofs for $\mathfrak{s o}(k)$ and $\mathfrak{s p}(\ell)$. By detailing the arguments for the $\operatorname{cases} \mathfrak{s o}(k), \mathfrak{s p}(\ell), \operatorname{or} \mathfrak{s u}(\ell+1)$, we also provide prototypes for the general, classification-free proofs in Section III - We start with the classification of simple alternating squares.

Theorem 1 (Dynkin). Let $\phi$ denote a faithful representation of a compact semisimple Lie algebra $\mathfrak{g}$ such that the alternating square $\mathrm{Alt}^{2} \phi$ is simple. All possible cases (up to outer automorphisms of $\mathfrak{g}$ ) are given in Table $\mathbb{\square}$. 
TABLE I. Irreducible representations whose respective alternating square is also irreducible (Dynkin)

\begin{tabular}{|c|c|c|c|c|c|c|}
\hline case & $\mathfrak{g}$ & $\ell$ & $\phi$ & $\operatorname{dim}(\phi)$ & $\operatorname{Alt}^{2} \phi$ & $\operatorname{dim}\left(\mathrm{Alt}^{2} \phi\right)$ \\
\hline (1a) & $\mathfrak{s o}(2 \ell+1)$ & $\ell>2$ & $(1,0, \ldots, 0)$ & $2 \ell+1$ & $(0,1,0, \ldots, 0)$ & $(2 \ell+1) \ell$ \\
\hline (1b) & $\mathfrak{s o}(5)$ & - & $(1,0)$ & 5 & $(0,2)$ & 10 \\
\hline$(2 a)$ & $\mathfrak{s o}(2 \ell)$ & $\ell>3$ & $(1,0, \ldots, 0)$ & $2 \ell$ & $(0,1,0, \ldots, 0)$ & $(2 \ell-1) \ell$ \\
\hline$(2 \mathrm{~b})$ & $\mathfrak{s o}(6)$ & - & $(1,0,0)$ & 6 & $(0,1,1)$ & 15 \\
\hline (3) & $\mathfrak{s u}(\ell+1)$ & $\ell \geq 3$ & $(0,1,0, \ldots, 0)$ & $\frac{\ell(\ell+1)}{2}$ & $(1,0,1,0, \ldots, 0)$ & $3\left(\begin{array}{c}\ell+2 \\
4\end{array}\right)$ \\
\hline$(4 a)$ & $\mathfrak{s u}(\ell+1)$ & $\ell>1$ & $(2,0, \ldots, 0)$ & $\frac{(\ell+1)(\ell+2)}{2}$ & $(2,1,0, \ldots, 0)$ & $3\left(\begin{array}{c}\ell+3 \\
4\end{array}\right)$ \\
\hline$(4 b)$ & $\mathfrak{s u}(2)$ & - & $(2)$ & 3 & $(2)$ & 3 \\
\hline (5) & $\mathfrak{s o}(10)$ & - & $(0,0,0,1,0)$ & 16 & $(0,0,1,0,0)$ & 120 \\
\hline (6) & $\mathfrak{e}_{6}$ & - & $(1,0,0,0,0,0)$ & 27 & $(0,0,1,0,0,0)$ & 351 \\
\hline (7a) & $\mathfrak{s u}(\ell+1)$ & $\ell>1$ & $(1,0, \ldots, 0)$ & $\ell+1$ & $(0,1,0, \ldots, 0)$ & $\frac{\ell(\ell+1)}{2}$ \\
\hline$(7 b)$ & $\mathfrak{s u}(2)$ & - & $(1)$ & 2 & $(0)$ & 1 \\
\hline
\end{tabular}

TABLE II. Irreducible representations whose respective symmetric square is also irreducible

\begin{tabular}{lllllll}
\hline \hline case & $\mathfrak{g}$ & $\ell$ & $\phi$ & $\operatorname{dim}(\phi)$ & $\operatorname{Sym}^{2} \phi$ & $(2,0, \ldots, 0)$ \\
\hline$(1)$ & $\mathfrak{s p}(\ell)$ & $\ell \geq 1$ & $(1,0, \ldots, 0)$ & $2 \ell$ & $(2 \ell+1) \ell$ \\
$(2)$ & $\mathfrak{s u}(\ell+1)$ & $\ell \geq 1$ & $(1,0, \ldots, 0)$ & $\ell+1$ & $(2,0, \ldots, 0)$ & $\frac{(\ell+1)(\ell+2)}{2}$ \\
\hline \hline
\end{tabular}

A streamlined proof of Theorem 1 is given in Appendix C 1. This result implies the following theorem for distinguishing $\mathfrak{s o}(k)$ with $k \geq 5$ from its subalgebras (see also Thm. 15 in Ref. 15), but we also provide now a simplified proof relying on ideas from the proof of Theorem 36 in Appendix C.

Theorem 2. Given a subalgebra $\mathfrak{h}$ of $\mathfrak{s o}(k)$ with $k \geq 5$ and the standard representation $\phi:=\phi_{(1,0, \ldots, 0)}$ of $\mathfrak{s o}(k)$, the following statements are equivalent:

(a) $\mathfrak{h}=\mathfrak{s o}(k)$.

(b) The representation $\left.\left(\mathrm{Alt}^{2} \phi\right)\right|_{\mathfrak{h}}$ is simple.

(c) The representation $\left.\left(\mathrm{Alt}^{2} \phi\right)\right|_{\mathfrak{h}}$ is simple and $\left.\left(\mathrm{Sym}^{2} \phi\right)\right|_{\mathfrak{h}}$ splits into two simple components. No simple component occurs more than once.

(d) The representation $\left.(\phi \otimes \phi)\right|_{\mathfrak{h}}$ splits into three different simple components.

(e) The vector space of all complex matrices commuting with $\left.(\phi \otimes \phi)\right|_{\mathfrak{h}}$ has dimension three.

Proof. Note that $\mathrm{Alt}^{2} \phi$ is equivalent to the adjoint representation and is simple (see Lemma 35 of Appendix B). In particular, $\mathrm{Alt}^{2} \phi=\phi_{(0,2)}$ for $\mathfrak{s o}(5), \mathrm{Alt}^{2} \phi=\phi_{(0,1,1)}$ for $\mathfrak{s o}(6)$, and $\mathrm{Alt}^{2} \phi=\phi_{(0,1,0, \ldots, 0)}$ for $k \geq 7$. We have $\operatorname{Sym}^{2} \phi=\phi_{(2,0, \ldots, 0)} \oplus \phi_{(0, \ldots, 0)}$ (see Ex. 19.21 of Ref. 16). It follows that both (b) and (c) are a consequence of (a). Obviously, (b) follows from (c). The adjoint representation $\mathrm{Alt}^{2} \phi$ is no longer simple when restricted to a proper subalgebra (see Lemma 35 of Appendix B) and (b) implies (a). The statements (c) and (d) are equivalent as $\left.\left(\mathrm{Sym}^{2} \phi\right)\right|_{\mathfrak{h}}$ splits into at least two components. The equivalence of (d) and (e) follows from Lemma 21 of Appendix $\mathrm{A}$.

Note that the proof relies critically on the irreducibility of the adjoint representation of $\mathfrak{s o}(k)$ for $k \geq 5$. - We present now the classification of simple symmetric squares.

Theorem 3. Let $\phi$ denote a faithful representation of a compact semisimple Lie algebra $\mathfrak{g}$ such that the symmetric square $\mathrm{Sym}^{2} \phi$ is simple. All possible cases (up to outer automorphisms of $\mathfrak{g}$ ) are given in Table II.

A streamlined proof of Theorem 3 is given in Appendix C2. This result implies the following theorem for distinguishing $\mathfrak{s p}(\ell)$ with $\ell \geq 2$ from its subalgebras, but we also provide now a simplified proof relying on ideas from the proof of Theorem 39 in Appendix C

Theorem 4. Given a subalgebra $\mathfrak{h}$ of $\mathfrak{s p}(\ell)$ with $\ell \geq 2$ and the standard representation $\phi:=\phi_{(1,0, \ldots, 0)}$ of $\mathfrak{s p}(\ell)$, the following statements are equivalent: 
(a) $\mathfrak{h}=\mathfrak{s p}(\ell)$.

(b) The representation $\left.\left(\mathrm{Sym}^{2} \phi\right)\right|_{\mathfrak{h}}$ is simple.

(c) The representation $\left.\left(\mathrm{Sym}^{2} \phi\right)\right|_{\mathfrak{h}}$ is simple and $\left.\left(\mathrm{Alt}^{2} \phi\right)\right|_{\mathfrak{h}}$ splits into two simple components. No simple component occurs more than once.

(d) The representation $\left.(\phi \otimes \phi)\right|_{\mathfrak{h}}$ splits into three different simple components.

(e) The vector space of all complex matrices commuting with $\left.(\phi \otimes \phi)\right|_{\mathfrak{h}}$ has dimension three.

Proof. Note that $\operatorname{Sym}^{2} \phi=\phi_{(2,0, \ldots, 0)}$ is equivalent to the adjoint representation and is always simple (see Lemma 35 of Appendix B). We have $\operatorname{Alt}^{2} \phi=\phi_{(0,1,0, \ldots, 0)} \oplus \phi_{(0, \ldots, 0)}$ which follows from the discussion on pp. 259-262 of Ref. 16 or pp. 206-209 of Ref. 17. We obtain that (b) and (c) are a consequence of (a). Obviously, (b) follows from (c). If $\mathfrak{h}$ is a proper subalgebra of $\mathfrak{s p}(\ell)$, the adjoint representation $\operatorname{Sym}^{2} \phi$ is no longer simple when restricted to $\mathfrak{h}$ (see Lemma 35 of Appendix B). Thus, (b) implies (a). The statements (c) and (d) are equivalent as $\left.\left(\operatorname{Alt}^{2} \phi\right)\right|_{\mathfrak{h}}$ splits into at least two components. The equivalence of (d) and (e) follows from Lemma 21 of Appendix A.

We emphasize that the proof applies the irreducibility of the adjoint representation of $\mathfrak{s p}(\ell)$ for $\ell \geq 2$.- Combining Theorems 1 and 3 we obtain a second proof of the classification of representations for which both the alternating square and the symmetric square are simple (see Ref. 4).

Theorem 5 (see Thm. 54 of Ref. (4). Let $\phi$ denote a faithful representation of a compact semisimple Lie algebra $\mathfrak{g}$ such that both the alternating square $\mathrm{Alt}^{2} \phi$ and the symmetric square $\mathrm{Sym}^{2} \phi$ are simple. Then $\mathfrak{g}=\mathfrak{s u}(\ell+1)$ with $\ell \geq 1$ and $\phi$ is (up to outer automorphisms of $\mathfrak{g}$ ) the standard representation with highest weight $(1,0, \ldots, 0)$.

The classification culminates into a convenient necessary and sufficient condition for deciding if a subalgebra of $\mathfrak{s u}(\ell+1)$ is proper (which also proves Theorem $\mathrm{A}$ ).

Theorem 6 (see Thm. 21 of Ref. 4). Given a subalgebra $\mathfrak{h}$ of $\mathfrak{s u}(\ell+1)$ with $\ell \geq 1$ and the standard representation $\phi:=\phi_{(1,0, \ldots, 0)}$ of $\mathfrak{s u}(\ell+1)$, the following statements are equivalent:

(a) $\mathfrak{h}=\mathfrak{s u}(\ell+1)$.

(b) The representations $\left.\left(\mathrm{Alt}^{2} \phi\right)\right|_{\mathfrak{h}}$ and $\left.\left(\mathrm{Sym}^{2} \phi\right)\right|_{\mathfrak{h}}$ are simple.

(c) The representations $\left.\left(\mathrm{Alt}^{2} \phi\right)\right|_{\mathfrak{h}}$ and $\left.\left(\mathrm{Sym}^{2} \phi\right)\right|_{\mathfrak{h}}$ are simple. No simple component occurs more than once.

(d) The representation $\left.(\phi \otimes \phi)\right|_{\mathfrak{h}}$ splits into two different simple components.

(e) $\operatorname{dim}\left(\operatorname{com}\left[\left.(\phi \otimes \phi)\right|_{\mathfrak{h}}\right]\right)=2$.

Proof. Statement (b) follows from (a) due to Theorem 5. We apply Lemma 24(iii) of Appendix @ to statement (b) and obtain that $\left.\phi\right|_{\mathfrak{h}}$ is simple. Therefore, the commutant of $\phi(\mathfrak{h})$ is trivial, i.e., it is equal to complex multiples of the identity. We conclude that the centralizer of $\phi(\mathfrak{h})$ in $\phi[\mathfrak{s u}(\ell+1)]$ is zero and the center of $\mathfrak{h}$ is also zero. Thus, $\mathfrak{h}$ is semisimple and we can use Theorem 5 to prove (a). Obviously, (b) and (c) are equivalent as $\left.\left(\mathrm{Alt}^{2} \phi\right)\right|_{\mathfrak{h}}$ and $\left.\left(\mathrm{Sym}^{2} \phi\right)\right|_{\mathfrak{h}}$ differ if both are simple. The statements (c) and (d) follow from each other as $\left.(\phi \otimes \phi)\right|_{\mathfrak{h}}=\left.\left.\left(\operatorname{Alt}^{2} \phi\right)\right|_{\mathfrak{h}} \oplus\left(\operatorname{Sym}^{2} \phi\right)\right|_{\mathfrak{h}}$. The equivalence of (d) and (e) is a consequence of Lemma 21 in Appendix A.

\section{CLASSIFICATION-FREE RESULTS}

Building on the approach of Section III, we develop now classification-free methods leading to general results for distinguishing compact semisimple Lie algebras from its subalgebras. This will in particular provide a proof for Theorem B. We start by introducing and discussing one- and two- "norms" in Section ЏII and continue by relating $\phi \otimes \psi$ to $\phi \otimes \bar{\psi}$ for semisimple representations $\phi$ and $\psi$ of a compact Lie algebra (see Section [IIB). In Section [IIC] we apply properties of the adjoint representation in order to prove the central result of Theorem 14 We summarize our classification-free results in Section [IID by presenting a set of statements which are equivalent to $\mathfrak{h}=\mathfrak{g}$ for a subalgebra $\mathfrak{h}$ of a compact semisimple Lie algebra $\mathfrak{g}$.

\section{A. One- and two-"norms"}

We consider a semisimple representation $\phi$ of a compact Lie algebra $\mathfrak{g}$. The decomposition of $\phi$ into simple representations can be given in the form $\oplus_{i \in \mathcal{I}}\left[\mathbb{1}_{m_{i}} \otimes \phi_{i}\right]$ of Lemma 21] in Appendix $\mathbb{A}$ where $m_{i}$ denotes the corresponding multiplicity. In the following, we will use the more concise notation $\oplus_{i \in \mathcal{I}} \phi_{i}^{\oplus m_{i}}$. It will be convenient to introduce the notations $\|\phi\|_{1}:=\sum_{i \in \mathcal{I}} m_{i}$ and $\|\phi\|_{2}:=\sum_{i \in \mathcal{I}} m_{i}^{2}$. We have as an immediate consequence of Lemma 21 in Appendix A that $\|\phi\|_{2}$ is equal to the dimension of the commutant of $\phi(\mathfrak{g})$. Moreover, we obtain the following propositions. 
Proposition 7. Consider two semisimple representations $\phi \cong \oplus_{i \in \mathcal{I}} \phi_{i}^{\oplus m_{i}}$ and $\psi \cong \oplus_{i \in \mathcal{I}} \psi_{i}^{\oplus n_{i}}$ of a compact Lie algebra $\mathfrak{g}$ which decomposes into simple representations $\phi_{i}$ and $\psi_{i}$ with multiplicities $m_{i}$ and $n_{i}$, respectively. One obtains (i) $\|\phi \oplus \psi\|_{1}=\|\phi\|_{1}+\|\psi\|_{1}$ and (ii) $\|\phi \oplus \psi\|_{2} \geq\|\phi\|_{2}+\|\psi\|_{2}$.

Proof. Statement (i) follows from $\|\phi \oplus \psi\|_{1}=\sum_{i \in \mathcal{I}} m_{i}+n_{i}=\|\phi\|_{1}+\|\psi\|_{1}$. Similarly, (ii) is a consequence of $\|\phi \oplus \psi\|_{2}=$ $\sum_{i \in \mathcal{I}}\left(m_{i}+n_{i}\right)^{2}=\sum_{i \in \mathcal{I}} m_{i}^{2}+2 m_{i} n_{i}+n_{i}^{2} \geq \sum_{i \in \mathcal{I}} m_{i}^{2}+n_{i}^{2}=\|\phi\|_{2}+\|\psi\|_{2}$.

Proposition 8. Consider a semisimple representation $\phi \cong \oplus_{i \in \mathcal{I}} \phi_{i}^{\oplus m_{i}}$ of a compact Lie algebra $\mathfrak{g}$ which decomposes into simple representations $\phi_{i}$ with multiplicities $m_{i}$. The restrictions of $\phi$ and $\phi_{i}$ to a subalgebra $\mathfrak{h}$ of $\mathfrak{g}$ are given by $\left.\phi\right|_{\mathfrak{h}} \cong \oplus_{j \in \mathcal{J}} \psi_{j}^{\oplus n_{j}}$ and $\left.\left(\phi_{i}\right)\right|_{\mathfrak{h}} \cong \oplus_{j \in \mathcal{J}} \psi_{j}^{\oplus n_{j i}}$ where $\psi_{j}$ denotes a simple representation of $\mathfrak{h}$ and $n_{j}=\sum_{i \in \mathcal{I}} n_{j i} m_{i}$. We obtain

(a) $\left\|\left.\left(\phi_{i}\right)\right|_{\mathfrak{h}}\right\|_{1}=1$ for all $i \in \mathcal{I}$ with $m_{i} \neq 0$ if and only if $\left\|\left.\phi\right|_{\mathfrak{h}}\right\|_{1}=\|\phi\|_{1}$,

(b) $\left\|\left.\phi\right|_{\mathfrak{h}}\right\|_{2}=\|\phi\|_{2}$ implies $\left\|\left.\phi\right|_{\mathfrak{h}}\right\|_{1}=\|\phi\|_{1}$,

(c) $\left\|\left.\phi\right|_{\mathfrak{h}}\right\|_{2}=\|\phi\|_{2} \Leftrightarrow\left\|\left.\phi\right|_{\mathfrak{h}}\right\|_{1}=\|\phi\|_{1}$ and $\left.\left(\phi_{i}\right)\right|_{\mathfrak{h}} \neq\left.\left(\phi_{k}\right)\right|_{\mathfrak{h}}$ holds for all $i, k \in \mathcal{I}$ with $i \neq k, m_{i} \neq 0$, and $m_{k} \neq 0$.

Proof. We assume during the proof that $\mathcal{I}$ contains only elements $i$ with $m_{i} \neq 0$. Note that $\|\phi\|_{1}=\sum_{i \in \mathcal{I}} m_{i},\left\|\left.\left(\phi_{i}\right)\right|_{\mathfrak{h}}\right\|_{1}=$ $\sum_{j \in \mathcal{J}} n_{j i}$, and $\left\|\left.\phi\right|_{\mathfrak{h}}\right\|_{1}=\sum_{j \in \mathcal{J}} n_{j}=\sum_{j \in \mathcal{J}}\left(\sum_{i \in \mathcal{I}} n_{j i} m_{i}\right)=\sum_{i \in \mathcal{I}}\left(\sum_{j \in \mathcal{J}} n_{j i}\right) m_{i}$. Furthermore, $\left\|\left.\phi\right|_{\mathfrak{h}}\right\|_{1}=\|\phi\|_{1}$ if and only if $\sum_{j \in \mathcal{J}} n_{j i}=1$ for all $i \in \mathcal{I}$ if and only if $\left\|\left.\left(\phi_{i}\right)\right|_{\mathfrak{h}}\right\|_{1}=1$ for all $i \in \mathcal{I}$. This completes the proof of (a). We remark that $\|\phi\|_{2}=\sum_{i \in \mathcal{I}} m_{i}^{2}$ and use the multinomial theorem to obtain

$$
\begin{aligned}
\left\|\left.\phi\right|_{\mathfrak{h}}\right\|_{2} & =\sum_{j \in \mathcal{J}} n_{j}^{2}=\sum_{j \in \mathcal{J}}\left(\sum_{i \in \mathcal{I}} n_{j i} m_{i}\right)^{2}=\sum_{j \in \mathcal{J}}\left(2 \sum_{\substack{i, \ell \in \mathcal{I} \\
i \neq \ell}} n_{j i} m_{i} n_{j \ell} m_{\ell}+\sum_{i \in \mathcal{I}} n_{j i}^{2} m_{i}^{2}\right) \\
& =2 \sum_{\substack{i, \ell \in \mathcal{I} \\
i \neq \ell}}\left(\sum_{j \in \mathcal{J}} n_{j i} n_{j \ell}\right) m_{i} m_{\ell}+\sum_{i \in \mathcal{I}}\left(\sum_{j \in \mathcal{J}} n_{j i}^{2}\right) m_{i}^{2} .
\end{aligned}
$$

Note that $\sum_{j \in \mathcal{J}} n_{j i}^{2} \geq 1$ for all $i \in \mathcal{I}$. We get from $\left\|\left.\phi\right|_{\mathfrak{h}}\right\|_{2}=\|\phi\|_{2}$ that (i) $\sum_{j \in \mathcal{J}} n_{j i}^{2}=1$ for all $i \in \mathcal{I}$ and that (ii) $\sum_{j \in \mathcal{J}} n_{j i} n_{j \ell}=0$ for all $i, \ell \in \mathcal{I}$ with $i \neq \ell$. Condition (i) implies that $\sum_{j \in \mathcal{J}} n_{j i}=1$ holds for all $i \in \mathcal{I}$. We can now prove (b) by applying (a) to the fact that $\left\|\left.\left(\phi_{i}\right)\right|_{\mathfrak{h}}\right\|_{1}=\sum_{j \in \mathcal{J}} n_{j i}=1$ is valid for all $i \in \mathcal{I}$. We consider now the statement (c). The fact that the condition $\left.(*)\left(\phi_{i}\right)\right|_{\mathfrak{h}} \neq\left.\left(\phi_{\ell}\right)\right|_{\mathfrak{h}}$ holds for all $i, \ell \in \mathcal{I}$ with $i \neq \ell$ is implied by (i) and (ii). This completes the direction " $\Rightarrow$ ". It follows (i) from $\left\|\left.\phi\right|_{\mathfrak{h}}\right\|_{1}=\|\phi\|_{1}$ by applying (a). The conditions (i) and (*) imply (ii) and the conditions (i) and (ii) imply $\left\|\left.\phi\right|_{\mathfrak{h}}\right\|_{2}=\|\phi\|_{2}$. This completes the direction " $\Leftarrow "$.

Note that the converse of part (b) in Proposition 8 is in general false.

\section{B. From $\phi \otimes \psi$ to $\phi \otimes \bar{\psi}$}

Here, we provide a Lie-algebraic argument why $\|\phi \otimes \psi\|_{2}=\|\phi \otimes \bar{\psi}\|_{2}$ holds for semisimple representations $\phi$ and $\psi$ of a compact Lie algebra.

Proposition 9. Given a compact Lie algebra $\mathfrak{g}$ and two semisimple representations $\phi$ and $\psi$ of $\mathfrak{g}$, it follows that $\mathfrak{c o m}(\phi \otimes \psi)=\mathbb{1} \otimes \tau[\mathfrak{c o m}(\phi \otimes \bar{\psi})]$, where $\tau$ denotes the transpose operation (acting on the second tensor component).

Proof. Let us recall that the dual representation is given by $\bar{\phi}(g):=-\phi(g)^{T}$ for $g \in \mathfrak{g}$, hence $\phi \otimes \bar{\psi}(g)=\phi(g) \otimes$ $\mathbb{1}-\mathbb{1} \otimes \psi(g)^{T}$. Suppose that $\sum_{i} v_{i} \otimes w_{i} \in \mathfrak{c o m}(\phi \otimes \psi)$, i.e., for any $g \in \mathfrak{g}$ one has $\left[(\phi \otimes \psi)(g), \sum_{i} v_{i} \otimes w_{i}\right]=0$. Considering the commutator of the element $\mathbb{1} \otimes \tau\left(\sum_{i} v_{i} \otimes w_{i}\right)=\sum_{i} v_{i} \otimes w_{i}^{T}$ with $(\phi \otimes \bar{\psi})(g)$, we arrive at the relation $\left[\sum_{i} v_{i} \otimes w_{i}^{T},(\phi \otimes \bar{\psi})(g)\right]=\sum_{i}\left[v_{i}, \phi(g)\right] \otimes w_{i}^{T}-\sum_{i} v_{i} \otimes\left[w_{i}^{T}, \psi(g)^{T}\right]=\sum_{i}\left[v_{i}, \phi(g)\right] \otimes w_{i}^{T}+\sum_{i} v_{i} \otimes\left[w_{i}, \psi(g)\right]^{T}=\mathbb{1} \otimes$ $\tau\left(\left[\sum_{i} v_{i} \otimes w_{i},(\phi \otimes \psi)(g)\right)\right]=\mathbb{1} \otimes \tau(0)=0$, thus $(\mathbb{1} \otimes \tau)\left(\sum_{i} v_{i} \otimes w_{i}\right) \in \mathfrak{c o m}(\phi \otimes \bar{\psi})$. Completely analogously one can prove that for any $\sum p_{i} \otimes q_{i} \in \mathfrak{c o m}(\phi \otimes \bar{\psi})$ one has that $\mathbb{1} \otimes \tau^{-1}\left(\sum_{i} p_{i} \otimes q_{i}\right)=\mathbb{1} \otimes \tau\left(\sum_{i} p_{i} \otimes q_{i}\right) \in \mathfrak{c o m}(\phi \otimes \psi)$. This completes the proof.

Proposition 9 can be readily applied in the proof of the following proposition.

Proposition 10. Given a compact Lie algebra $\mathfrak{g}$ and two semisimple representations $\phi$ and $\psi$ of $\mathfrak{g}$, it follows that $\|\phi \otimes \psi\|_{2}=\|\phi \otimes \bar{\psi}\|_{2}$.

Proof. According to Lemma 21 of Appendix $\mathrm{A}$, it follows that $\|\phi \otimes \psi\|_{2}=\operatorname{dim} \mathfrak{c o m}(\phi \otimes \psi)$ and $\|\phi \otimes \bar{\psi}\|_{2}=\operatorname{dim} \operatorname{com}(\phi \otimes \bar{\psi})$. From Proposition 9 we know that $\mathfrak{c o m}(\phi \otimes \psi)$ is mapped by a non-degenerate linear map (the partial transpose) to $\mathfrak{c o m}(\phi \otimes \bar{\psi})$, so the dimensions of the two commutants are equal, thus also $\|\phi \otimes \psi\|_{2}$ and $\|\phi \otimes \bar{\psi}\|_{2}$ are equal. 


\section{Using the adjoint representation}

The adjoint representation plays a important part in our argument, and we recall and develop now some of its properties in order to prove our central result of Theorem [14 as given below.

Proposition 11. Consider a compact semisimple Lie algebra $\mathfrak{g}$ and its decomposition $\mathfrak{g} \cong \oplus_{i \in \mathcal{I}} \mathfrak{g}_{i}$ into simple ideals $\mathfrak{g}_{i}$. (a) The adjoint representation $\theta_{\mathfrak{g}}$ of $\mathfrak{g}$ decomposes as $\theta_{\mathfrak{g}} \cong \oplus_{i \in \mathcal{I}} \theta_{\mathfrak{g}_{i}}$. (b) It is simple if $\mathfrak{g}$ is simple. (c) The adjoint representation $\left.\left(\theta_{\mathfrak{g}}\right)\right|_{\mathfrak{h}}$ of $\mathfrak{g}$ restricted to a proper subalgebra $\mathfrak{h}$ is reducible. (d) The adjoint representation $\theta_{\mathfrak{h}}$ of $\mathfrak{h}$ occurs as a subrepresentation of $\left.\left(\theta_{\mathfrak{g}}\right)\right|_{\mathfrak{h}}$.

Proof. The statement (a) is apparent. The statements (b) and (c) follow from Lemma 35 of Appendix B. The adjoint representation $\theta_{\mathfrak{g}}$ of $\mathfrak{g}$ constitutes an action $\mathfrak{g} \times \mathfrak{g} \rightarrow \mathfrak{g}$ which is defined using the commutator $\left[g_{1}, g_{2}\right]=g_{3}$ for $g_{i}$ in $\mathfrak{g}$. If one restricts $\theta_{\mathfrak{g}}$ to elements of $\mathfrak{h}$, the representation $\left.\left(\theta_{\mathfrak{g}}\right)\right|_{\mathfrak{h}}$ forms an action $\mathfrak{h} \times \mathfrak{g} \rightarrow \mathfrak{g}$ by $\left[h, g_{2}\right]=g_{3}$ with $h \in \mathfrak{h}$ and $g_{i}$ in $\mathfrak{g}$. The adjoint representation $\theta_{\mathfrak{h}}$ occurs as a subrepresentation as $\left[h_{1}, h_{2}\right] \in \mathfrak{h}$ for $h_{i} \in \mathfrak{h}$. Statement (d) follows.

This immediately implies the following result.

Proposition 12. Given a subalgebra $\mathfrak{h}$ of a compact semisimple Lie algebra $\mathfrak{g}$ and their adjoint representations $\theta_{\mathfrak{h}}$ and $\theta_{\mathfrak{g}}$, then $\mathfrak{h}=\mathfrak{g}$ if and only if $\left\|\left.\left(\theta_{\mathfrak{g}}\right)\right|_{\mathfrak{h}}\right\|_{1}=\left\|\theta_{\mathfrak{g}}\right\|_{1}$.

Proof. One has to show that $\left\|\left.\left(\theta_{\mathfrak{g}}\right)\right|_{\mathfrak{h}}\right\|_{1} \neq\left\|\theta_{\mathfrak{g}}\right\|_{1}$ if $\mathfrak{h} \neq \mathfrak{g}$. Assuming that $\mathfrak{h} \neq \mathfrak{g}$, Proposition 11(c) implies that $\left.\left(\theta_{\mathfrak{g}}\right)\right|_{\mathfrak{h}}$ has more simple components than $\theta_{\mathfrak{g}}$. It follows that $\left\|\left.\left(\theta_{\mathfrak{g}}\right)\right|_{\mathfrak{h}}\right\|_{1}>\left\|\theta_{\mathfrak{g}}\right\|_{1}$ which concludes the proof.

Let us recall some well-known connection between the standard and the adjoint representation of $\mathfrak{s u}(\ell+1)$.

Proposition 13. Given the standard representation $\kappa$ of $\mathfrak{s u}(\ell+1)$, one obtains $\kappa \otimes \bar{\kappa} \cong 1 \oplus \theta_{\mathfrak{s u}(\ell+1)}$, where 1 denotes the trivial representation and $\theta_{\mathfrak{s u}(\ell+1)}$ denotes the adjoint representation of $\mathfrak{s u}(\ell+1)$.

Proof. For $\mathfrak{s u}(\ell+1)$, the standard representation $\kappa$, the dual $\bar{\kappa}$ of $\kappa$, the adjoint representation $\theta_{\mathfrak{s u}(\ell+1)}$, and the trivial representation have highest weights $(1,0, \ldots, 0),(0, \ldots, 0,1),(1,0, \ldots, 0,1)$ (and $(2)$ for $\ell=1)$, and $(0, \ldots, 0)$, respectively. The proposition can now be inferred from the statements on p. 225 of Ref. 16.

We can now combine all previous results in this section in order to prove the following central theorem.

Theorem 14. Given a subalgebra $\mathfrak{h}$ of a compact semisimple Lie algebra $\mathfrak{g}$ and a faithful representation $\phi$ of $\mathfrak{g}$, then $\mathfrak{h}=\mathfrak{g}$ if and only if $\left\|\left.(\phi \otimes \bar{\phi})\right|_{\mathfrak{h}}\right\|_{1}=\|\phi \otimes \bar{\phi}\|_{1}$.

Proof. Let $d:=\operatorname{dim}(\phi)$. As $\phi$ is faithful, $\mathfrak{g} \subseteq \mathfrak{s u}(d)$. Given the standard reprepresentation $\kappa$ of $\mathfrak{s u}(d)$, we obtain $\phi=\left.\kappa\right|_{\mathfrak{g}}$ and $\phi \otimes \bar{\phi}=\left.\left.\kappa\right|_{\mathfrak{g}} \otimes \bar{\kappa}\right|_{\mathfrak{g}}=\left.(\kappa \otimes \bar{\kappa})\right|_{\mathfrak{g}}$. Using Propositions 13 and 11(d) it follows that $\theta_{\mathfrak{s u}(d)}$ occurs in $\kappa \otimes \bar{\kappa}$ and $\theta_{\mathfrak{g}}$ occurs in $\left.\theta_{\mathfrak{s u}(d)}\right|_{\mathfrak{g}}$. Therefore, $\theta_{\mathfrak{g}}$ occurs in $\phi \otimes \bar{\phi}$. But $\theta_{\mathfrak{g}}$ splits when restricted to $\mathfrak{h} \neq \mathfrak{g}$ [see Proposition 11 (c)] and it follows that $\left\|\left.\left(\theta_{\mathfrak{g}}\right)\right|_{\mathfrak{h}}\right\|_{1}>\left\|\theta_{\mathfrak{g}}\right\|_{1}$ [see Proposition 12. We apply Proposition 8 (a) and conclude that there exists a simple representation in the decomposition of $\theta_{\mathfrak{g}}$ which splits when restricted to $\mathfrak{h}$. But this simple representation also appears in the decomposition of $\phi \otimes \bar{\phi}$, and we can apply Proposition 8 (a) again to conclude that $\left\|\left.(\phi \otimes \bar{\phi})\right|_{\mathfrak{h}}\right\|_{1}>\|\phi \otimes \bar{\phi}\|_{1}$.

\section{Classification-free theorem}

Recall that $\mathfrak{c o m}[\phi]$ denotes the commutant of a representation $\phi$ of a Lie algebra $\mathfrak{g}$. We summarize our results in a convenient omnibus theorem which relates the equality of a compact semisimple Lie algebra $\mathfrak{g}$ to one of its subalgebras $\mathfrak{h}$ to the condition $\operatorname{dim}\left(\mathfrak{c o m}\left[\left.(\phi \otimes \phi)\right|_{\mathfrak{h}}\right]\right)=\operatorname{dim}(\mathfrak{c o m}[\phi \otimes \phi])$ for a faithful representation $\phi$ of $\mathfrak{g}$ (and thereby also proving Theorem B), as well as to various other variants.

Theorem 15. Given a subalgebra $\mathfrak{h}$ of a compact semisimple Lie algebra $\mathfrak{g}$ and a faithful representation $\phi$ of $\mathfrak{g}$, then the following statements are equivalent:

(1) $\mathfrak{h}=\mathfrak{g}$,

(2) $\operatorname{dim}\left(\operatorname{com}\left[\left.(\phi \otimes \bar{\phi})\right|_{\mathfrak{h}}\right]\right)=\operatorname{dim}(\mathfrak{c o m}[\phi \otimes \bar{\phi}])$,

(3) $\operatorname{dim}\left(\operatorname{com}\left[\left.(\phi \otimes \phi)\right|_{\mathfrak{h}}\right]\right)=\operatorname{dim}(\operatorname{com}[\phi \otimes \phi])$,

(4) $\left\|\left.(\phi \otimes \bar{\phi})\right|_{\mathfrak{h}}\right\|_{2}=\|\phi \otimes \bar{\phi}\|_{2}$,

(5) $\left\|\left.(\phi \otimes \bar{\phi})\right|_{\mathfrak{h}}\right\|_{1}=\|\phi \otimes \bar{\phi}\|_{1}$,

(6) $\left\|\left.(\phi \otimes \phi)\right|_{\mathfrak{h}}\right\|_{2}=\|\phi \otimes \phi\|_{2}$.

(7) $\left\|\left.(\phi \otimes \phi)\right|_{\mathfrak{h}}\right\|_{1}=\|\phi \otimes \phi\|_{1}$. 
Note that the proof of the equivalence of (7) relies on a theorem of Coquereaux and Zuber (see Appendix (D) for which a classification-free proof is (to our knowledge) not yet known.

Proof. Obviously, statement (1) implies all the other ones. The equivalence of (2) and (4) as well as (3) and (6) is a consequence of Lemma 21 in Appendix A. One obtains the equivalence of (4) and (6) by applying Proposition 10. Proposition 8(b) shows that (5) is implied by (4). It remains to prove that (5) implies (1) which follows by Theorem 14. Note that (5) is equivalent to (7) by applying Proposition 46 from Appendix D.

\section{DISCUSSION}

It is natural to ask if and how one could extend the results of Theorem 15 to general compact Lie algebras. A first step in this direction is given by the following theorem which allows us to conclude that the semisimple parts of $\mathfrak{h}$ and $\mathfrak{g}$ have to be equal if the dimensions of the commutant of the tensor squares are equal assuming that the corresponding representation is both faithful and semisimple.

Theorem 16. Consider a subalgebra $\mathfrak{h}$ of a compact Lie algebra $\mathfrak{g}=\mathfrak{s}(\mathfrak{g}) \oplus \mathfrak{c}(\mathfrak{g})$ (which decomposes into its semisimple part $\mathfrak{s}(\mathfrak{g})$ and its center $\mathfrak{c}(\mathfrak{g})$ ) as well as a faithful and semisimple representation $\phi$ of $\mathfrak{g}$. One has $\mathfrak{s}(\mathfrak{h})=\mathfrak{s}(\mathfrak{g})$ if $\operatorname{dim}\left(\mathfrak{c o m}\left[\left.(\phi \otimes \phi)\right|_{\mathfrak{h}}\right]\right)=\operatorname{dim}(\mathfrak{c o m}[\phi \otimes \phi])$.

The corresponding proof is given in Appendix E It is important to emphasize that the converse of Theorem [16 is in general not true as the following counter-example shows: Consider the two Lie-algebra generators $A$ and $B$ which are given in a faithful representation $\phi$ as commuting matrices

$$
\phi(A):=\left(\begin{array}{ccc}
1 & 0 & 0 \\
0 & 1 & 0 \\
0 & 0 & -2
\end{array}\right) \text { and } \phi(B):=\left(\begin{array}{ccc}
2 & 0 & 0 \\
0 & -1 & 0 \\
0 & 0 & -1
\end{array}\right) .
$$

Note that $A$ generates a one-dimensional abelian Lie algebra $\mathfrak{h}$ isomorphic to $\mathfrak{u}(1)$ and that $A$ and $B$ generate a two-dimensional abelian Lie algebra $\mathfrak{g}$ isomorphic to $\mathfrak{u}(1) \oplus \mathfrak{u}(1)$. Their semisimple parts $\mathfrak{s}(\mathfrak{h})=\mathfrak{s}(\mathfrak{g})=\{0\}$ are equal and trivial, but $\operatorname{dim}\left(\mathfrak{c o m}\left[\left.(\phi \otimes \phi)\right|_{\mathfrak{h}}\right]\right)=33$ and $\operatorname{dim}\left(\mathfrak{c o m}\left[\left.(\phi \otimes \phi)\right|_{\mathfrak{g}}\right]\right)=15$. In order to treat the case of a general compact Lie algebra completely, an additional condition for guaranteeing the equality of the centers of $\mathfrak{h}$ and $\mathfrak{g}$ will be necessary. This is the topic of a related study $\frac{18}{18}$ which focuses on control-theoretic applications in quantum systems.

A different possibility of extending our results in Theorem[15 is by providing not only inequalities as $\left\|\left.(\phi \otimes \phi)\right|_{\mathfrak{h}}\right\|_{1}>$ $\|\phi \otimes \phi\|_{1}$ and $\left\|\left.(\phi \otimes \phi)\right|_{\mathfrak{h}}\right\|_{2}>\|\phi \otimes \phi\|_{2}$. But one can in special cases determine bounds on the gap in these inequalities. If $\mathfrak{g}$ is simple and $\phi$ is self-dual, one can apply a result of King and Wybourne $\frac{19}{}$ in order to derive the following bounds (see Appendix E).

Proposition 17. Let $\alpha$ be a simple and self-dual representation of a compact simple Lie algebra $\mathfrak{g}$, and let $\mathfrak{h}$ be a subalgebra of $\mathfrak{g}$, then

(1) $\left\|\left.(\alpha \otimes \alpha)\right|_{\mathfrak{h}}\right\|_{1} \geq b(\alpha)+\|\alpha \otimes \alpha\|_{1}$,

(2) $\left\|\left.(\alpha \otimes \alpha)\right|_{\mathfrak{h}}\right\|_{2} \geq b(\alpha)^{2}+\|\alpha \otimes \alpha\|_{2}$, and

(3) $\operatorname{dim}\left(\mathfrak{c o m}\left[\left.(\alpha \otimes \alpha)\right|_{\mathfrak{h}}\right]\right) \geq b(\alpha)^{2}+\operatorname{dim}(\mathfrak{c o m}[\alpha \otimes \alpha])$ hold,

where $b(\alpha)$ denotes the number of non-vanishing components in the highest weight $\left(\alpha_{1}, \ldots, \alpha_{\ell}\right)$ corresponding to $\alpha$.

Using the structures of $\mathfrak{g}$ and its representation $\phi$, it would be interesting to derive bounds which are better or which are applicable in other cases.

\section{ACKNOWLEDGMENTS}

R.Z. acknowledges support from the Deutsche Forschungsgemeinschaft (DFG) in the collaborative research center SFB 631 as well as from the EU programmes SIQS and QUAINT. Z.Z. acknowledges support from the British Engineering and Physical Sciences Research Council (EPSRC).

\section{Appendix A: Preliminaries}

In this appendix, we recall some basic facts about compact Lie algebras and their representations. Connections to the complexifications are highlighted in Appendix A1. This is followed by the well-known classification of simple 
representations into symplectic, orthogonal, and unitary ones. The definition and discussion of alternating and symmetric squares are given in Appendix A2.

\section{Compact Lie Algebras and their Representations}

Any compact (real) Lie algebra can be written as a direct sum of two compact Lie algebras where one is semisimple and the other one is commutative (see Chap. IX, Sec. 1.3, Prop. 1 of Ref. 17 and Chap. I, Sec. 6.4, Prop. 5 of Ref 20). The semisimple Lie algebra can be further decomposed into a direct sum of compact, simple Lie algebras which consists of the classical ones $\mathfrak{s u}(\ell+1), \mathfrak{s o}(2 \ell+1), \mathfrak{s p}(\ell):=\mathfrak{s p}(2 \ell, \mathbb{C}) \cap \mathfrak{s u}(2 \ell)$, and $\mathfrak{s o}(2 \ell)$ where $\ell \in \mathbb{N} \backslash\{0\}($ excluding $\mathfrak{s o}(2)$ and $\mathfrak{s o}(4) \cong \mathfrak{s u}(2) \oplus \mathfrak{s u}(2)$ which are not simple) as well as the exceptional ones $\mathfrak{g}_{2}, \mathfrak{f}_{4}, \mathfrak{e}_{6}, \mathfrak{e}_{7}, \mathfrak{e}_{8}($ see Ref. 17$)$. Note the isomorphisms $\mathfrak{s u}(2) \cong \mathfrak{s o}(3) \cong \mathfrak{s p}(1), \mathfrak{s o}(5) \cong \mathfrak{s p}(2)$, and $\mathfrak{s u}(4) \cong \mathfrak{s o}(6)$ (see, e.g., Thm. X.3.12 in Ref. 21).

We briefly recall some elementary facts about the complexification of a real Lie algebra which will be directly applied to compact Lie algebras. Let $\phi$ denote a (finite-dimensional) complex matrix representation of a real Lie algebra $\mathfrak{k}$, where $\phi$ maps a Lie-algebra element $k \in \mathfrak{k}$ to a square matrix $\phi(k) \in \mathfrak{g l}(d, \mathbb{C})$ of finite $\operatorname{degree} d:=\operatorname{dim}(\phi)$, where $\mathfrak{g l}(d, \mathbb{C})$ denotes the set of complex $d \times d$-matrices. All representations in this manuscript are matrix representations with complex matrix entries. For every real Lie algebra $\mathfrak{k}$, a representation $\phi$ of $\mathfrak{k}$ in $\mathfrak{g l}(d, \mathbb{C})$ can be naturally extended to a representation $\phi_{\mathbb{C}}$ of $\mathfrak{k}_{\mathbb{C}}$ in $\mathfrak{g l}(d, \mathbb{C})$, where $\mathfrak{k}_{\mathbb{C}}:=\mathfrak{k}+i \mathfrak{k}$ is the complexification of $\mathfrak{k}$.

Lemma 18. Consider a real Lie algebra $\mathfrak{k}$ and its complexification $\mathfrak{k}_{\mathbb{C}}$. Let $\tau$ denote a representation of $\mathfrak{k}_{\mathbb{C}}$. Moreover, let $\phi$ and $\psi$ denote two representations of $\mathfrak{k}$, and $\phi_{\mathbb{C}}$ and $\psi_{\mathbb{C}}$ the corresponding representations of $\mathfrak{k}_{\mathbb{C}}$. The following statements hold:

(i) The representations $\phi$ and $\psi$ are isomorphic if and only if the representations $\phi_{\mathbb{C}}$ and $\psi_{\mathbb{C}}$ are isomorphic.

(ii) For the representation $\tau$ of $\mathfrak{k}_{\mathbb{C}}$, there exists a representation $\eta$ of $\mathfrak{k}$ such that $\tau=\eta_{\mathbb{C}}$.

(iii) $\phi$ is simple (i.e., irreducible) if and only if $\phi_{\mathbb{C}}$ is simple.

(iv) $\phi$ is semisimple (i.e., completely reducible) if and only if $\phi_{\mathbb{C}}$ is semisimple.

(v) If two real Lie algebras are isomorphic, then so are their complexifications.

(vi) $\mathfrak{k}$ is abelian if and only if $\mathfrak{k}_{\mathbb{C}}$ is abelian.

(vii) $\mathfrak{k}$ is simple if $\mathfrak{k}_{\mathbb{C}}$ is simple.

(viii) $\mathfrak{k}$ is semisimple if and only if $\mathfrak{k}_{\mathbb{C}}$ is semisimple.

(ix) $\mathfrak{k}$ is reductive if and only if $\mathfrak{k}_{\mathbb{C}}$ is reductive.

Proof. Part (i) is trivial. In order to prove (ii), we set $\eta:=\left.\tau\right|_{\mathfrak{k}}$. The $\mathbb{R}$-linearity of $\eta$ follows from the $\mathbb{C}$-linearity of $\tau$. We obtain $\eta_{\mathbb{C}}\left(\mathfrak{k}_{\mathbb{C}}\right)=c_{1} \eta(\mathfrak{k})+c_{2} \eta(\mathfrak{k})=c_{1}(\tau \mid \mathfrak{k})(\mathfrak{k})+c_{2}\left(\left.\tau\right|_{\mathfrak{k}}\right)(\mathfrak{k})=c_{1} \tau(\mathfrak{k})+c_{2} \tau(\mathfrak{k})=\tau\left(c_{1} \mathfrak{k}+c_{2} \mathfrak{k}\right)=\tau(\mathfrak{k}+i \mathfrak{k})=\tau\left(\mathfrak{k}_{\mathbb{C}}\right)$ with $c_{j} \in \mathbb{C}$, and part (ii) follows. We address now part (iii): If $\phi$ is simple, so is $\phi_{\mathbb{C}}$. Assume that $\phi_{\mathbb{C}}$ is simple and $\phi$ is not simple. Thus, there exists two linear-independent complex matrices $M_{1}$ and $M_{2}$ which commute with $\phi(\mathfrak{k}), i \phi(\mathfrak{k})$, and $\phi(\mathfrak{k})+i \phi(\mathfrak{k})=\phi_{\mathbb{C}}\left(\mathfrak{k}_{\mathbb{C}}\right)$. This proves (iii) by contradiction. Given that $\phi$ is semisimple, we obtain that $\phi=\oplus_{k} \phi_{k}$ with $\phi_{k}$ simple. It follows that $\phi_{\mathbb{C}}$ is semisimple as it is isomorphic to $\oplus_{k}\left(\phi_{k}\right)_{\mathbb{C}}$. Assuming that $\phi_{\mathbb{C}}$ is semisimple, we have $\phi_{\mathbb{C}}=\oplus_{k} \tau_{k}$ where $\tau_{k}$ are simple representations of $\mathfrak{k}_{\mathbb{C}}$. Applying (ii) we obtain (complex) representations $\eta_{k}$ with $\tau_{k}=\left(\eta_{k}\right)_{\mathbb{C}}$. The representation $\phi$ is semisimple as it is isomorphic to $\oplus_{k} \eta_{k}$, which completes the proof of (iv). Assume that the real Lie algebras $\mathfrak{k}$ and $\mathfrak{k}^{\prime}$ are isomorphic. It follows that $\mathfrak{k}_{\mathbb{C}}=\mathfrak{k}+i \mathfrak{k} \cong \mathfrak{k}^{\prime}+i \mathfrak{k}^{\prime}=\mathfrak{k}_{\mathbb{C}}^{\prime}$ which proves $(\mathrm{v})$. For (vi)-(ix), we refer to Chap. I, Sec. 1.9 and Sec. 6.10 of Ref. 20.

We can sharpen Lemma 18 if we assume that the considered real Lie algebra is compact. Two compact Lie algebras $\mathfrak{g}$ and $\mathfrak{g}^{\prime}$ are isomorphic iff their complexifications $\mathfrak{g}_{\mathbb{C}}$ and $\mathfrak{g}_{\mathbb{C}}^{\prime}$ are isomorphic (see Cor. 1 of Thm. 1 in Chap. IX, Sec. 3.3 of Ref. 17). Referring to p. 283 and p. 300 of Ref. 17, we also obtain that a compact Lie algebra $\mathfrak{g}$ and its complexification $\mathfrak{g}_{\mathbb{C}}$ are reductive as well as that $\mathfrak{g}$ is simple (resp. semisimple) if and only if $\mathfrak{g}_{\mathbb{C}}$ is simple (resp. semisimple).

Lemma 19. Consider two compact Lie algebras $\mathfrak{g}$ and $\mathfrak{g}^{\prime}$ as well as their complexifications $\mathfrak{g}_{\mathbb{C}}$ and $\left(\mathfrak{g}^{\prime}\right) \mathbb{C}$. We obtain that (i) $\mathfrak{g}$ is isomorphic to $\mathfrak{g}^{\prime}$ iff $\mathfrak{g}_{\mathbb{C}}$ is isomorphic to $\left(\mathfrak{g}^{\prime}\right)_{\mathbb{C}}$ as well as (ii) $\mathfrak{g}$ is simple iff $\mathfrak{g}_{\mathbb{C}}$ is simple.

As a consequence of the previous discussion, certain questions about a compact Lie algebra $\mathfrak{g}$ (and its representations) will be answered by switching to its complexification $\mathfrak{g}_{\mathbb{C}}$ without further comment.-Although a compact Lie algebra might have a representation which is not semisimple (e.g., consider a one-dimensional Lie algebra with a representation which maps a Lie-algebra element $\lambda$ to the matrix $\left.\left(\begin{array}{ll}0 & 0 \\ \lambda & 0\end{array}\right)\right)$, any restriction of a semisimple representation to one of its ideals or subalgebras stays semisimple.

Lemma 20. Let us consider a semisimple (complex) representation $\phi$ of a compact Lie algebra $\mathfrak{g}$. The restriction $\left.\phi\right|_{\mathfrak{h}}$ of $\phi$ to any subalgebra $\mathfrak{h}$ of $\mathfrak{g}$ is still semisimple. 
TABLE III. Dynkin diagrams for the compact simple Lie algebras $[\mathfrak{s o}(2 \ell)$ is only simple for $\ell \geq 3]$

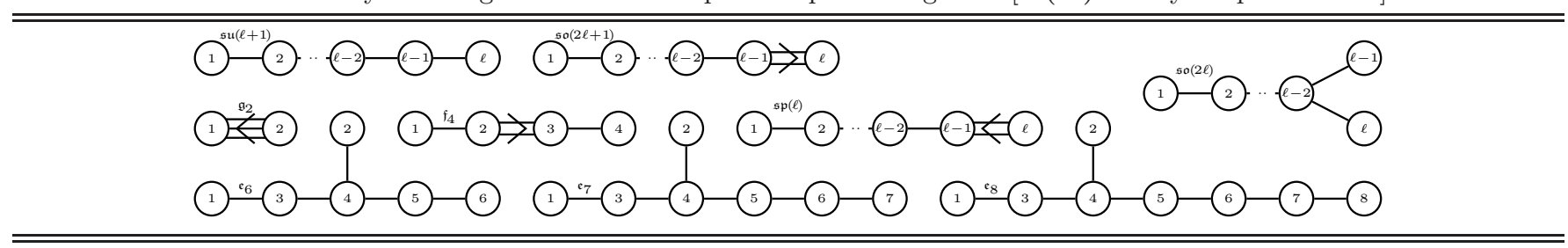

Proof. Note that the corresponding conclusion for ideals holds for arbitrary Lie algebras of characteristic zero, see Chap. I, Sec. 6.5, Cor. 4 to Thm. 4 of Ref. 20. In order to prove the statement, we apply that both $\mathfrak{g}$ and its subalgebra $\mathfrak{h}$ are compact and consequently reductive. We have $\mathfrak{h}=\mathfrak{h}_{s} \oplus \mathfrak{h}_{a}$ where $\mathfrak{h}_{s}$ is semsimple and $\mathfrak{h}_{a}$ is abelian. The representation $\left.\phi\right|_{\mathfrak{h}}$ is semsimple if and only if the set $\phi\left(\mathfrak{h}_{a}\right)$ contains only semisimple matrices (i.e., all elements of $\phi\left(\mathfrak{h}_{a}\right)$ are diagonalizable over $\mathbb{C}$ ), see Chap. I, Sec. 6.5, Thm. 4 of Ref. 20. We remark that $\mathfrak{h}_{a}$ is contained in a Cartan subalgebra $\mathfrak{t}_{\mathfrak{g}}$ of the compact Lie algebra $\mathfrak{g}$ as $\mathfrak{t}_{\mathfrak{g}}$ is a maximal abelian subalgebra (see Chap. IX, Sec. 2.1, Thm. 1 of Ref. 17). In particular, $\mathfrak{t}_{\mathfrak{g}}$ is abelian and $\phi\left(\mathfrak{t}_{\mathfrak{g}}\right)$ contains only semisimple matrices if $\phi$ is semisimple and $\mathfrak{g}$ is reductive (see Chap. VII, Sec. 2.4, Cor. 3 to Thm. 2 of Ref. 17). Thus, $\phi\left(\mathfrak{h}_{a}\right)$ also contains only semisimple matrices which completes the proof.

We also state some elementary consequences for the representations of compact Lie algebras from the representation theory of compact groups (see Theorem 1.5 of Ref. 22).

Lemma 21. Let $\phi$ denote a semisimple (complex) representation of a compact Lie algebra $\mathfrak{g}$. The degree of $\phi$ is given by $d:=\operatorname{dim}(\phi)$. The commutant algebra $\operatorname{com}(\phi)$ is defined as all complex $d \times d$-matrices which simultaneously commute with $\phi(g)$ for all $g \in \mathfrak{g}$. We obtain that $\phi$ is equivalent to $\oplus_{i \in \mathcal{I}}\left[\mathbb{1}_{m_{i}} \otimes \phi_{i}\right]$ where the distinct inequivalent simple representations $\phi_{i}$ of $\mathfrak{g}$ have degree $d_{i}$ and occur with multiplicity $m_{i}$ in the decomposition of $\phi$. In particular, (a) $\operatorname{dim} \mathfrak{c o m}(\phi)=\sum_{i \in \mathcal{I}} m_{i}^{2}$, (b) $\operatorname{dim} \mathfrak{c e n t e r}(\mathfrak{c o m}(\phi))=|\mathcal{I}|=\sum_{i \in \mathcal{I}, m_{i} \neq 0} 1$, and (c) $d=\sum_{i \in \mathcal{I}} d_{i} m_{i}$.

Recall that a bilinear form $B$ on $\mathbb{C}^{\operatorname{dim}(\phi)}$ is denoted as $\phi(\mathfrak{g})$-invariant iff $B\left(\phi(g) v_{1}, v_{2}\right)=-B\left(v_{1}, \phi(g) v_{2}\right)$ for all $g \in \mathfrak{g}$ and $v_{i} \in \mathbb{C}^{\operatorname{dim}(\phi)}$. Let $\mathcal{B}_{\phi}$ denote the vector space of $\phi(\mathfrak{g})$-invariant bilinear forms. The dual representation $\bar{\phi}$ is given as $\bar{\phi}(g):=-\phi(g)^{T}$ where $g \in \mathfrak{g}$ (see Chap. I, Sec. 3.3 of Ref 20 ); note that $\bar{\phi}(g)=-\phi(g)^{T}=\overline{\phi(g)}$ if $\phi(g)$ is skew-hermitian. We call a representation self-dual if it is equivalent to its dual representation. Assuming that $\phi$ is simple and self-dual, it follows that $\operatorname{dim}\left(\mathcal{B}_{\phi}\right)=1$ and every nonzero element of $\mathcal{B}_{\phi}$ is nondegenerate (see Chap. VIII, Sec. 7.5 and App. II.2 of Ref. 17 or Lemma 13 in Ref. 4 ). A self-dual, simple representation $\phi$ is called either orthogonal or symplectic depending on whether the corresponding $\phi(\mathfrak{g})$-invariant, nondegenerate bilinear form is symmetric or alternating (i.e., skew-symmetric), respectively. A simple representation $\phi$ of $\mathfrak{g}$ is orthogonal [or symplectic] iff $\phi(\mathfrak{g})$ conjugate to a subalgebra of $\mathfrak{s o}(\operatorname{dim}(\phi))[\operatorname{or} \mathfrak{s p}(\operatorname{dim}(\phi) / 2)]$ (cf. p. 336 of Ref. 1 or Thm. H on p. 144 of Ref. 23). If $\phi$ is simple but not self-dual, then no nondegenerate bilinear form is $\phi(\mathfrak{g})$-invariant and we denote $\phi$ as unitary. The orthogonal, symplectic, and unitary representations are also referred to as representations of real, quaternionic, or complex type, respectively.

A simple representation of a compact semisimple Lie algebra $\mathfrak{g}$ can be further characterized using the theory of the highest weight: 17 the highest weight is an integer vector $\left(x_{1}, \ldots, x_{\ell}\right)$ with $x_{j} \geq 0$ and identifies classes of simple representations where the coefficient $x_{j}$ indicates the contribution of the $j$-th fundamental weight (see Chap. VIII, Sec. 7.2 of Ref. 17). The coefficients are ordered according to a convention of Bourbaki (see Plate I-IX of Ref. 24 or Table III). The length $\ell$ is equal to the rank of $\mathfrak{g}$ which is defined as the dimension of any maximal abelian subalgebra of $\mathfrak{g}$. Using the notation of the highest weight, simple representations of compact, simple Lie algebras can be explicitly classified as symplectic, orthogonal, or unitary by the work of Malcev $\underline{25}$ (see Refs. 1, 23, 26, and 27):

Proposition 22 (Malcev). A simple representation $\phi$ of a compact simple Lie algebra $\mathfrak{g}$ is either symplectic, orthogonal, or unitary depending on its highest weight $x=\left(x_{1}, \ldots, x_{\ell}\right)$ : (1) Assuming that $\mathfrak{g} \cong \mathfrak{s u}(\ell+1)$, $\phi$ is unitary iff $x$ is not symmetric and symplectic iff $x$ is symmetric, $(\ell \bmod 4)=1$, and $x_{((\ell-1) \operatorname{div} 2)+1}$ is odd; it is orthogonal in all other cases. (2) In the case of $\mathfrak{g} \cong \mathfrak{s o}(2 \ell+1)$ with $\ell \geq 3$, we have that $\phi$ is symplectic iff $(\ell \bmod 4) \in\{1,2\}$ and $x_{\ell}$ is odd, while it is orthogonal otherwise. (3) For $\mathfrak{g}=\mathfrak{s p}(\ell)$ with $\ell \geq 2$, $\phi$ is symplectic if $\sum_{1 \leq 2 j+1 \leq \ell} x_{2 j+1}$ is odd for $j \in \mathbb{N} \cup\{0\}$, while it is orthogonal otherwise. (4) Assuming that $\mathfrak{g}=\mathfrak{s o}(2 \ell)$ for $\ell \geq 3$, (a) $\phi$ is symplectic if $(\ell \bmod 4)=2$ and $x_{\ell-1}+x_{\ell}$ is odd, (b) $\phi$ is orthogonal if either (i) $(\ell \bmod 4)=2$ and $x_{\ell-1}+x_{\ell}$ is even, (ii) $(\ell \bmod 4)=0$, or (iii) $\ell$ is odd and $x_{\ell-1}=x_{\ell}$, and $(c) \phi$ is unitary if $\ell$ is odd and $x_{\ell-1} \neq x_{\ell}$. (5) The representation $\phi$ is always orthogonal for $\mathfrak{g} \in\left\{\mathfrak{g}_{2}, \mathfrak{f}_{4}, \mathfrak{e}_{8}\right\}$. (6) Assuming that $\mathfrak{g} \cong \mathfrak{e}_{6}, \phi$ is orthogonal iff $x_{1}=x_{6}$ and $x_{3}=x_{5}$; it is unitary in all other cases. (7) For $\mathfrak{g} \cong \mathfrak{e}_{7}, \phi$ is symplectic iff $x_{2}+x_{5}+x_{7}$ is odd and orthogonal otherwise. 


\section{Alternating and Symmetric Square}

The (inner) tensor product $\phi \otimes \psi$ of two representations $\phi$ and $\psi$ of a compact Lie algebra $\mathfrak{g}$ is defined as $(\phi \otimes \psi)(g):=$ $\phi(g) \otimes \mathbb{1}_{\operatorname{dim}(\psi)}+\mathbb{1}_{\operatorname{dim}(\phi)} \otimes \psi(g)$ for $g \in \mathfrak{g}$, or more explicitly as $(\phi \otimes \psi)(g)\left[v_{i} \otimes w_{j}\right]:=\left[\phi(g) v_{i}\right] \otimes w_{j}+v_{i} \otimes\left[\psi(g) w_{j}\right]$ when acting on a basis $\left\{v_{i} \otimes w_{j} \mid i \in\{1, \ldots, \operatorname{dim}(\phi)\}\right.$ and $\left.j \in\{1, \ldots, \operatorname{dim}(\psi)\}\right\}$ of $\mathbb{C}^{\operatorname{dim}(\phi)} \otimes \mathbb{C}^{\operatorname{dim}(\psi)}$ (cf. Chap. I, Sec. 3.2 of Ref. 20). Similarly, we define the tensor square $\phi^{\otimes 2}(g):=(\phi \otimes \phi)(g)=\phi(g) \otimes \mathbb{1}_{\operatorname{dim}(\phi)}+\mathbb{1}_{\operatorname{dim}(\phi)} \otimes \phi(g)$ and the tensor power $\phi^{\otimes k}(g):=\phi(g) \otimes \mathbb{1}_{\operatorname{dim}\left(\phi^{\otimes k-1}\right)}+\mathbb{1}_{\operatorname{dim}(\phi)} \otimes \phi^{\otimes k-1}(g)$. This parallels the case of groups where the tensor product of representations is directly given by the Kronecker product of the representation matrices.

Let $\phi$ denote a $d$-dimensional representation of a compact Lie algebra $\mathfrak{g}$ acting on the vector space $V:=\mathbb{C}^{d}$ with basis $\left\{v_{i} \mid i \in\{1, \ldots, d\}\right\}$. Recall that $V^{\otimes 2}=V \otimes V$ decomposes into $\operatorname{Alt}^{2} V \oplus \operatorname{Sym}^{2} V$ where $\operatorname{Alt}^{2} V$ and $\operatorname{Sym}^{2} V$ are spanned by $\left\{v_{i} \otimes v_{j}-v_{j} \otimes v_{i} \mid i, j \in\{1, \ldots, d\}\right.$ and $\left.i \neq j\right\}$ and $\left\{v_{i} \otimes v_{j}+v_{j} \otimes v_{i} \mid i, j \in\{1, \ldots, d\}\right\}$, respectively. The alternating square $\mathrm{Alt}^{2} \phi:=\left.\phi^{\otimes 2}\right|_{\mathrm{Alt}^{2} V}$ of $\phi$ is defined by restricting $\phi^{\otimes 2}$ to $\operatorname{Alt}^{2} V$. Accordingly, the symmetric square $\operatorname{Sym}^{2} \phi$ is given by $\left.\phi^{\otimes 2}\right|_{\mathrm{Sym}^{2} V}$. The corresponding actions on basis vectors are

$$
\begin{aligned}
\left(\operatorname{Alt}^{2} \phi\right)(g)\left[v_{i} \otimes v_{j}-v_{j} \otimes v_{i}\right]= & \left(\left[\phi(g) v_{i}\right] \otimes v_{j}-v_{j} \otimes\left[\phi(g) v_{i}\right]\right) \\
& +\left(v_{i} \otimes\left[\phi(g) v_{j}\right]-\left[\phi(g) v_{j}\right] \otimes v_{i}\right) \text { and } \\
\left(\operatorname{Sym}^{2} \phi\right)(g)\left[v_{i} \otimes v_{j}+v_{j} \otimes v_{i}\right]= & \left(\left[\phi(g) v_{i}\right] \otimes v_{j}+v_{j} \otimes\left[\phi(g) v_{i}\right]\right) \\
& +\left(v_{i} \otimes\left[\phi(g) v_{j}\right]+\left[\phi(g) v_{j}\right] \otimes v_{i}\right) .
\end{aligned}
$$

Note that $\operatorname{dim}\left(\operatorname{Alt}^{2} \phi\right)=\operatorname{dim}\left(\operatorname{Alt}^{2} V\right)=d(d-1) / 2$ and $\operatorname{dim}\left(\operatorname{Sym}^{2} \phi\right)=\operatorname{dim}\left(\operatorname{Sym}^{2} V\right)=d(d+1) / 2$. In summary, we obtain the decomposition $\phi^{\otimes 2}=\phi \otimes \phi=\operatorname{Alt}^{2} \phi \oplus \operatorname{Sym}^{2} \phi$. First, we recall one elementary lemma.

Lemma 23. Consider two compact Lie algebras $\mathfrak{h}$ and $\mathfrak{g}$ with $\mathfrak{h} \subseteq \mathfrak{g}$ as well as a faithful representation $\phi$ of $\mathfrak{g}$. We obtain $\left.\left(\phi^{\otimes 2}\right)\right|_{\mathfrak{h}}=\left(\left.\phi\right|_{\mathfrak{h}}\right)^{\otimes 2},\left.\left(\operatorname{Alt}^{2} \phi\right)\right|_{\mathfrak{h}} \cong \operatorname{Alt}^{2}\left(\left.\phi\right|_{\mathfrak{h}}\right)$, and $\left.\left(\operatorname{Sym}^{2} \phi\right)\right|_{\mathfrak{h}} \cong \operatorname{Sym}^{2}\left(\left.\phi\right|_{\mathfrak{h}}\right)$.

Proof. We have $\left.\left((\{\phi(g) \mid g \in \mathfrak{g}\})^{\otimes 2}\right)\right|_{\mathfrak{h}}=\left.(\{\phi(g) \otimes \mathbb{1}+\mathbb{1} \otimes \phi(g) \mid g \in \mathfrak{g}\})\right|_{\mathfrak{h}}=\{\phi(g) \otimes \mathbb{1}+\mathbb{1} \otimes \phi(g) \mid g \in \mathfrak{h}\}=\left(\left.(\{\phi(g) \mid g \in \mathfrak{g}\})\right|_{\mathfrak{h}}\right)^{\otimes 2}$. The rest follows along the same lines.

Another elementary consequence of the definitions is the following lemma.

Lemma 24. Let $\phi$ and $\psi$ denote two representations of a compact Lie algebra $\mathfrak{g}$.

(i) $\operatorname{Alt}^{2}(\phi \oplus \psi)=\operatorname{Alt}^{2} \phi \oplus(\phi \otimes \psi) \oplus \operatorname{Alt}^{2} \psi$,

(ii) $\operatorname{Sym}^{2}(\phi \oplus \psi)=\operatorname{Sym}^{2} \phi \oplus(\phi \otimes \psi) \oplus \operatorname{Sym}^{2} \psi$,

(iii) $\phi$ is simple if $\mathrm{Sym}^{2} \phi$ is simple, and

(iv) $\phi$ is simple if $\mathrm{Alt}^{2} \phi$ is simple, $\phi$ is faithful, and $\mathfrak{g}$ is semisimple.

Proof. Statements (iii) and (iv) are a consequence of (i) and (ii) which both can be found on p. 473 of Ref. 16.

Let $\phi_{1}$ and $\phi_{2}$ denote representations of the Lie algebras $\mathfrak{g}_{1}$ and $\mathfrak{g}_{2}$, respectively. The outer tensor product $\phi_{1} \otimes \phi_{2}$ is defined as $(\phi \otimes \psi)\left(g_{1}, g_{2}\right):=\phi_{1}\left(g_{1}\right) \otimes \mathbb{1}_{\operatorname{dim}\left(\phi_{2}\right)}+\mathbb{1}_{\operatorname{dim}\left(\phi_{1}\right)} \otimes \phi_{2}\left(g_{2}\right)$ with $g_{1} \in \mathfrak{g}_{1}$ and $g_{2} \in \mathfrak{g}_{2}$. The statement of the following lemma can be deduced from pp. 48-49 of Ref. 28 (see also p. 69 of Ref. 29 ).

Lemma 25. Assume that $\phi_{i}$ is a representation of a compact Lie algebra $\mathfrak{g}_{i}$ where $i \in\{1,2\}$.

(i) $\operatorname{Alt}^{2}\left(\phi_{1} \otimes \phi_{2}\right)=\left(\mathrm{Sym}^{2} \phi_{1} \otimes \operatorname{Alt}^{2} \phi_{2}\right) \oplus\left(\operatorname{Alt}^{2} \phi_{1} \otimes \operatorname{Sym}^{2} \phi_{2}\right)$,

(ii) $\operatorname{Sym}^{2}\left(\phi_{1} \otimes \phi_{2}\right)=\left(\operatorname{Sym}^{2} \phi_{1} \otimes \operatorname{Sym}^{2} \phi_{2}\right) \oplus\left(\operatorname{Alt}^{2} \phi_{1} \otimes \operatorname{Alt}^{2} \phi_{2}\right)$.

A compact Lie algebra $\mathfrak{g}$ can be decomposed as $\mathfrak{g} \cong \oplus_{k} \mathfrak{g}_{k}$ where $\mathfrak{g}_{k}$ denotes a simple or abelian ideal of $\mathfrak{g}$. Moreover, all simple representations of $\mathfrak{g}$ are of the form $\otimes_{k} \phi_{k}$ where $\phi_{k}$ denotes a simple representation of $\mathfrak{g}_{k}$.

Lemma 26. Consider a compact Lie algebra $\mathfrak{g} \cong \mathfrak{g}_{1} \oplus \mathfrak{g}_{2}$. All finite-dimensional simple representations $\phi$ of $\mathfrak{g}$ are of the form $\phi_{1} \otimes \phi_{2}$ where $\phi_{k}$ denotes simple representations of $\mathfrak{g}_{k}$. Furthermore, $\phi_{1} \otimes \phi_{2}$ is a simple representation of $\mathfrak{g}$ for any simple representations $\phi_{k}$ of $\mathfrak{g}_{k}$.

Proof. This follows directly from the corresponding fact for (finite-dimensional) representations over $\mathbb{C}$ of unital, associative algebras over $\mathbb{C}$, see Sect. 12.1 in Ref. 30.

Recall that the trivial representation maps every element to the zero matrix of degree one, which is the only onedimensional representation of semisimple Lie algebras. We obtain as a consequence of Lemmas 24 26 the following theorem. 
TABLE IV. Cartan matrices $\alpha_{\mathfrak{g}}$ corresponding to the root system of $\mathfrak{g}_{\mathbb{C}}$

\begin{tabular}{|c|c|c|c|c|}
\hline $\begin{array}{l}\alpha_{\mathfrak{s u}(\ell+1)}= \\
\alpha_{\mathfrak{s o}(2 \ell)}=\end{array}$ & 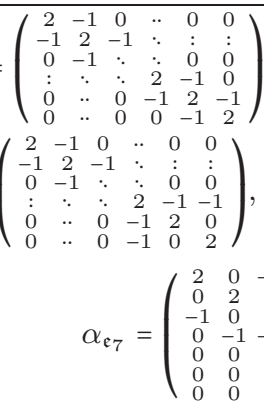 & 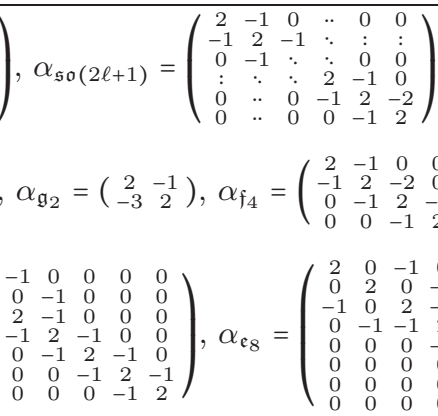 & $\begin{array}{ll}, \alpha_{\mathfrak{s p}(\ell)}=( \\
0 \\
0 \\
-1 \\
-1 \\
2\end{array}$ & $\left.\begin{array}{cccccc}2 & -1 & 0 & \because & 0 & 0 \\
-1 & 2 & -1 & \ddots & \vdots & \vdots \\
0 & -1 & \ddots & \ddots & 0 & 0 \\
\vdots & \ddots & \ddots & 2 & -1 & 0 \\
0 & \ddots & 0 & -1 & 2 & -1 \\
0 & - & 0 & 0 & -2 & 2 \\
2 & 0 & -1 & 0 & 0 & 0 \\
0 & 2 & 0 & -1 & 0 & 0 \\
-1 & 0 & 2 & -1 & 0 & 0 \\
0 & -1 & -1 & 2 & -1 & 0 \\
0 & 0 & 0 & -1 & 2 & -1 \\
0 & 0 & 0 & 0 & -1 & 2\end{array}\right)$, \\
\hline
\end{tabular}

Theorem 27. Let $\phi$ denote a faithful, semisimple representation of a compact Lie algebra $\mathfrak{g} \cong \mathfrak{s} \oplus \mathfrak{c}$ where $\mathfrak{s} \cong \oplus_{k} \mathfrak{s}_{k}$ is semisimple (or zero) and $\mathfrak{c}$ is abelian. We assume that $\mathrm{Alt}^{2} \phi$ or $\mathrm{Sym}^{2} \phi$ is simple. It follows that $\left.\phi\right|_{\mathfrak{s}}$ is simple, and $\left.\phi\right|_{\mathfrak{s}_{k}}$ is distinct from the trivial representation for at most one $k$. Moreover, the Lie algebra $\mathfrak{s}$ is simple (or zero).

Proof. As $\mathfrak{g}$ is compact, it is reductive and decomposes as $\mathfrak{g} \cong \mathfrak{s} \oplus \mathfrak{c}$ where $\mathfrak{s}$ is semisimple and $\mathfrak{c}$ is abelian. The representation $\left.\phi\right|_{\mathfrak{s}}$ is simple by Lemma 24. It follows from Lemma 26 that all simple representations of a direct sum of Lie algebras are given by outer tensor products of simple representations. Assuming that $\left.\phi\right|_{\mathfrak{s}_{k}}$ is distinct from the trivial representation for more than one $k$ results in a contradiction with Lemma 25. The remaining statement follows immediately, see also pp. 27-28 and p. 321 of Ref. 31 for information on irreducible subalgebras.

As the decomposition $\mathfrak{g} \cong \mathfrak{s} \oplus \mathfrak{c}$ of a compact (or reductive) Lie algebra will appear often, we recall that $[\mathfrak{g}, \mathfrak{g}]:=$ $\left\{\left[g_{1}, g_{2}\right] \mid g_{1}, g_{2} \in \mathfrak{g}\right\}$ denotes an ideal (and a subalgebra) of the Lie algebra $\mathfrak{g}$. If $\mathfrak{g}$ is reductive (or compact), [ $\left.\mathfrak{g}, \mathfrak{g}\right]$ is a semisimple subalgebra of $\mathfrak{g}$ and we denote it by $\mathfrak{s}(\mathfrak{g}):=[\mathfrak{g}, \mathfrak{g}]$. Moreover, the center of $\mathfrak{g}$ is given by $\mathfrak{c}(g):=\left\{g_{1} \in\right.$ $\mathfrak{g} \mid\left[g_{1}, g_{2}\right]=0$ for all $\left.g_{2} \in \mathfrak{g}\right\}$. We obtain that $\mathfrak{g}=\mathfrak{s}(\mathfrak{g}) \oplus \mathfrak{c}(\mathfrak{g})$ if $\mathfrak{g}$ is reductive (or compact), where $\mathfrak{s}=\mathfrak{s}(\mathfrak{g})$ and $\mathfrak{c}=\mathfrak{c}(\mathfrak{g})$. Theorem 27 can be used to simplify the discussion by limiting the search for simple, alternating and symmetric squares to simple representations of compact simple Lie algebras.

\section{Appendix B: Some techniques of Dynkin}

We recall some elementary but powerful techniques of Dynkin (see Sec. 3 of Ref. 1) which allow us to analyze the decomposition of a tensor product of representations very efficiently. In particular, we discuss minimal chains, the notion of subordination, and the method of parts. At the end of this appendix, some elementary facts about the adjoint representation are also recalled. Combinations of these techniques will be directly applied, e.g., in Appendix C.

As discussed in Appendix A1, the highest weight $x_{\phi}=\left(x_{\phi, 1}, \ldots, x_{\phi, \ell}\right)$ characterizes an equivalence class corresponding to a simple representation $\phi$ of a compact semisimple Lie algebra $\mathfrak{g}$ of rank $\ell$. The root system $R$ of $\mathfrak{g}_{\mathbb{C}}$ (see Chap. VIII, Sec. 2 of Ref. 17 and Chap. VI of Ref. 24) has a basis $\left\{\alpha_{1}, \ldots, \alpha_{\ell}\right\}$ of so-called simple roots $\alpha_{a}=\left(\alpha_{a, 1}, \ldots, \alpha_{a, \ell}\right)$ such that the corresponding Cartan matrix $\alpha_{\mathfrak{g}}$ has entries $\alpha_{a, b}$ (see Table IV). The Dynkin diagrams in Table III can be recovered from the Cartan matrices: there is a link between nodes $a$ and $b$ if $\alpha_{a, b} \neq 0$, and its type is then given by the value of $\alpha_{a, b} / \alpha_{b, a} \in\{1,2,3,1 / 2,1 / 3\}$ (see Chap. VI, Sec. 4.2 of Ref. 24). The basis of simple roots introduces a partial order (e.g.,) on the highest weights: $x_{\phi} \leq x_{\psi}$ if $x_{\psi}-x_{\phi}=\sum_{j=1}^{\ell} n_{j} \alpha_{j}$ for some non-negative integers $n_{j}$. Using this notation, one obtains the following lemma (see Chap. VIII, Sec. 7.4, Prop. 9(ii) of Ref. 17).

Lemma 28. Consider a compact semisimple Lie algebra $\mathfrak{g}$ and two of its simple representations $\phi$ and $\psi$. The tensor product $\phi \otimes \psi \cong \oplus_{k} \tau_{k}$ decomposes into simple representations $\tau_{k}$ with $x_{\tau_{k}} \leq x_{\phi}+x_{\psi}$

Let $(\cdot \cdot)$ denote a non-degenerate symmetric bilinear form on the real vector space generated by the root system $R$ where $\alpha_{a, b}=2\left(\alpha_{a} \mid \alpha_{b}\right) /\left(\alpha_{b} \mid \alpha_{b}\right)$ and $x_{a}=2\left(x \mid \alpha_{a}\right) /\left(\alpha_{a} \mid \alpha_{a}\right)$ (see pp. 157-158 and 180-181 of Ref. 24). The explicit form of (.|.) will not be important in the following, but we emphasize that $\left(\alpha_{a} \mid \alpha_{b}\right)=0 \Leftrightarrow \alpha_{a, b}=0 \Leftrightarrow \alpha_{b, a}=0$. Now, more detailed information on the tensor product decomposition can be obtained by applying the notion of a minimal chain linking two highest weights (see Sec. 3.1 of Ref. 1, Chap. VIII, Sec. 7, Ex. 18 of Ref. 17, or Ref. 32).

Definition 29. Consider two highest weights $x_{\phi}$ and $x_{\psi}$ corresponding to two simple representations $\phi$ and $\psi$ of a compact semisimple Lie algebra $\mathfrak{g}$. A sequence $\left[\alpha_{j_{1}}, \ldots, \alpha_{j_{n}}\right]$ of simple roots of $\mathfrak{g}_{\mathbb{C}}$ is said to be a chain joining $x_{\phi}$ and $x_{\psi}$ if (a) $\left(x_{\phi} \mid \alpha_{j_{1}}\right) \neq 0$, (b) $\left(\alpha_{j_{k}} \mid \alpha_{j_{k+1}}\right) \neq 0$ for $k \in\{1, \ldots, n-1\}$, and (c) $\left(\alpha_{j_{n}} \mid x_{\psi}\right) \neq 0$. Such a chain is called minimal 
if no proper subsequence joins $x_{\phi}$ and $x_{\psi}$, i.e., (d) $\left(x_{\phi} \mid \alpha_{j_{k}}\right)=0$ for $k>1$, (e) $\left(\alpha_{j_{k}} \mid \alpha_{j_{h}}\right)=0$ for $h>k+1$, and (f) $\left(\alpha_{j_{k}} \mid x_{\psi}\right)=0$ for $k<n$.

Using a minimal chain, we can restrict the possible highest weights in the decomposition of a tensor product.

Proposition 30. If $x_{\tau} \neq x_{\phi}+x_{\psi}$ is the highest weight of a simple representation $\tau$ occurring in the decomposition of the tensor product $\phi \otimes \psi$ of two simple representations $\phi$ and $\psi$, then $x_{\tau} \leq x_{\phi}+x_{\psi}-\sum_{k=1}^{n} \alpha_{j_{k}}$ for some minimal chain $\left[\alpha_{j_{1}}, \ldots, \alpha_{j_{n}}\right]$ linking $x_{\phi}$ and $x_{\psi}$. In particular, both $x_{\phi}+x_{\psi}-\sum_{k=1}^{n} \alpha_{j_{k}}$ and $x_{\phi}+x_{\psi}$ appear in the decomposition.

We follow Dynkin (Sec. 3.3 in Ref. 1) and introduce the powerful notion of subordination, see also Ref. 32 and Chap. VIII, Sec. 7, Ex. 16 of Ref. 17.

Definition 31. We consider the representations $\phi \cong \oplus_{j=1}^{r} \phi_{j}$ and $\psi \cong \oplus_{j=1}^{s} \psi_{j}$ of a compact simple Lie algebra which decompose into simple representations $\phi_{j}$ and $\psi_{j}$. Let $x_{\tau}=\left(x_{\tau, 1}, \ldots, x_{\tau, \ell}\right)$ denote the highest weight of a simple representation $\tau$. Assuming that both $\phi$ and $\psi$ are simple (i.e., $r=s=1$ ), $\phi$ is called subordinate to $\psi$ (written $\phi \sqsubseteq \psi$ or $\left.x_{\phi} \sqsubseteq x_{\psi}\right)$ if $x_{\phi, k} \leq x_{\psi, k}$ for all $k \in\{1, \ldots, \ell\}$. Otherwise, we call $\phi$ subordinate to $\psi$ if $r \leq s$ and there exists a re-ordering of the simple components such that $\phi_{j} \sqsubseteq \psi_{j}$ for $j \in\{1, \ldots, r\}$.

Important consequences of subordination are summarized in the following proposition where $m(\phi, \psi)$ (or $m\left(x_{\phi}, \psi\right)$ ) denotes the multiplicity of the simple representation $\phi$ in the decomposition of the representation $\psi$.

Proposition 32. Let $\phi, \phi_{j}, \psi$, and $\psi_{j}$ denote simple representations of a compact simple Lie algebra $\mathfrak{g}$ such that $\phi \sqsubseteq \psi$ and $\phi_{j} \sqsubseteq \psi_{j}$ for $j \in\{1,2\}$. Given a basis $\left\{\alpha_{1}, \ldots, \alpha_{\ell}\right\}$ of simple roots for $\mathfrak{g}_{\mathbb{C}}$ and a set $\left\{n_{1}, \ldots, n_{\ell}\right\}$ of non-negative integers, we obtain:

(i) $\phi_{1} \otimes \phi_{2} \sqsubseteq \psi_{1} \otimes \psi_{2}$.

(ii) $m\left(x_{\phi_{1}}+x_{\phi_{2}}-\sum_{k=1}^{\ell} n_{k} \alpha_{k}, \phi_{1} \otimes \phi_{2}\right) \leq m\left(x_{\psi_{1}}+x_{\psi_{2}}-\sum_{k=1}^{\ell} n_{k} \alpha_{k}, \psi_{1} \otimes \psi_{2}\right)$.

(iii) $m\left(2 x_{\phi}-\sum_{k=1}^{\ell} n_{k} \alpha_{k}, \operatorname{Sym}^{2} \phi\right) \leq m\left(2 x_{\psi}-\sum_{k=1}^{\ell} n_{k} \alpha_{k}, \operatorname{Sym}^{2} \psi\right)$.

(iv) $m\left(2 x_{\phi}-\sum_{k=1}^{\ell} n_{k} \alpha_{k}, \operatorname{Alt}^{2} \phi\right) \leq m\left(2 x_{\psi}-\sum_{k=1}^{\ell} n_{k} \alpha_{k}, \operatorname{Alt}^{2} \psi\right)$.

Proof. Part (i) is Thm. 3.17 of Ref. 1. A proof of the other parts is given in Thm. 1 of Ref. 32.

Consider the basis $\left\{\alpha_{1}, \ldots, \alpha_{\ell}\right\}$ of simple roots of $\mathfrak{g}_{\mathbb{C}}$ for a compact semisimple Lie algebra $\mathfrak{g}$. A proper subset of the simple roots obtained by deleting the simple roots $\alpha_{j}$ with $j \in D \mp\{1, \ldots, \ell\}$ generates a smaller root system corresponding to a semisimple subalgebra $\mathfrak{h}$ of $\mathfrak{g}$ (resp. $\mathfrak{h}_{\mathbb{C}}$ of $\mathfrak{g}_{\mathbb{C}}$ ), cf. Chap. VIII, Sec. 3.1 of Ref. 17. This is equivalent to deleting the nodes in $D$ from the Dynkin diagram or shortening the highest weight $x$ of a simple representation by deleting the entries $x_{j}$ with $j \in D$. This allows us to introduce the notion of parts of highest weights (see Sec. 3.2 of Ref. 1 and Chap. XIV of Ref. 33i).

Definition 33. Consider the representations $\phi \cong \oplus_{j=1}^{r} \phi_{j}$ and $\psi \cong \oplus_{j=1}^{s} \psi_{j}$ of the compact semsimple Lie algebras $\mathfrak{h}$ and $\mathfrak{g}$, respectively. In addition, $\phi_{j}$ and $\psi_{j}$ denote simple representations and $\mathfrak{f}$ is a subalgebra of $\mathfrak{g}$. Assuming that both $\phi$ and $\psi$ are simple (i.e., $r=s=1$ ), $\phi$ is called a part of $\psi$ (written $\phi \subseteq \psi$ or $x_{\phi} \subseteq x_{\psi}$ ) if $\phi$ is faithful and $x_{\phi}$ is a shortened version of $x_{\psi}$ which is obtained by deleting some (but not all) or none of the entries $x_{\psi, j}$. Otherwise, $\phi$ is a part of $\psi$ if $r \leq s$ and there exists a re-ordering of the simple components such that $\phi_{j} \subseteq \psi_{j}$ for $j \in\{1, \ldots, r\}$.

We obtain the following proposition which is essential in applying the method of parts.

Proposition 34. Consider the semisimple subalgebra $\mathfrak{h}$ of the compact semisimple Lie algebra $\mathfrak{g}$. Let $\phi$ and $\phi_{j}$ (resp. $\psi$ and $\psi_{j}$ ) denote simple representations of $\mathfrak{h}$ (resp. $\mathfrak{g}$ ). Assume that $\phi \subseteq \psi$ and $\phi_{j} \subseteq \psi_{j}$. Given a basis $\left\{\alpha_{1}, \ldots, \alpha_{\ell}\right\}$ of the simple roots of $\mathfrak{g}_{\mathbb{C}}$ and a set $\left\{n_{1}, \ldots, n_{\ell}\right\}$ of non-negative integers, we obtain:

(i) $\phi_{1} \otimes \phi_{2} \subseteq \psi_{1} \otimes \psi_{2}$.

(ii) $m\left(x_{\phi_{1}}+x_{\phi_{2}}-\sum_{k=1}^{\ell} n_{k} \alpha_{k}, \phi_{1} \otimes \phi_{2}\right) \leq m\left(x_{\psi_{1}}+x_{\psi_{2}}-\sum_{k=1}^{\ell} n_{k} \alpha_{k}, \psi_{1} \otimes \psi_{2}\right)$.

(iii) $m\left(2 x_{\phi}-\sum_{k=1}^{\ell} n_{k} \alpha_{k}, \operatorname{Sym}^{2} \phi\right) \leq m\left(2 x_{\psi}-\sum_{k=1}^{\ell} n_{k} \alpha_{k}, \operatorname{Sym}^{2} \psi\right)$.

(iv) $m\left(2 x_{\phi}-\sum_{k=1}^{\ell} n_{k} \alpha_{k}, \mathrm{Alt}^{2} \phi\right) \leq m\left(2 x_{\psi}-\sum_{k=1}^{\ell} n_{k} \alpha_{k}, \mathrm{Alt}^{2} \psi\right)$.

Proof. Part (i) is Thm. 3.10 of Ref. 1. Part (ii) follows from (i) and Lemma28 by applying Thms. 3.7 and 3.9 of Ref. 1 . We also recommend the discussion in Chap. XIV of Ref. 33. A proof of (ii)-(iv) is given in Thm. 1 of Ref. 34 .

Finally, recall that the adjoint representation of a Lie algebra $\mathfrak{g}$ maps each element $g_{1} \in \mathfrak{g}$ to the endomorphism $\operatorname{ad}_{\mathfrak{g}}\left(g_{1}\right)$ which is defined as $\left(\operatorname{ad}_{\mathfrak{g}}\left(g_{1}\right)\right)\left(g_{2}\right):=\left[g_{1}, g_{2}\right]$ for each $g_{2} \in \mathfrak{g}$. We summarize some facts about the adjoint representation of a simple Lie algebra. These results are essential for dealing with self-dual representations.

Lemma 35. Consider a compact real (or complex) Lie algebra $\mathfrak{g}$. (i) The adjoint representation of $\mathfrak{g}$ restricted to a proper subalgebra is reducible. (ii) The adjoint representation of $\mathfrak{g}$ is simple if $\mathfrak{g}$ is simple. (iii) The adjoint representation of $\mathfrak{s o}(k)$ with $k \geq 3$ is isomorphic to the alternating square of the standard representation; moreover 
$\operatorname{Alt}^{2} \phi_{(2)}=\phi_{(2)}$ for $\mathfrak{s o}(3) \cong \mathfrak{s u}(2), \operatorname{Alt}^{2} \phi_{(1,1)}=\phi_{(2,2)}$ for $\mathfrak{s o}(4), \operatorname{Alt}^{2} \phi_{(1,0)}=\phi_{(0,2)}$ for $\mathfrak{s o}(5), \operatorname{Alt}^{2} \phi_{(1,0,0)}=\phi_{(0,1,1)}$ for $\mathfrak{s o}(6)$, and $\mathrm{Alt}^{2} \phi_{(1,0, \ldots, 0)}=\phi_{(0,1,0, \ldots, 0)}$ for $k \geq 7$. (iv) The adjoint representation of $\mathfrak{s p}(\ell)$ with $\ell \geq 1$ is isomorphic to the symmetric square $\operatorname{Sym}^{2} \phi_{(1,0, \ldots, 0)}=\phi_{(2,0, \ldots, 0)}$ of the standard representation $\phi_{(1,0, \ldots, 0)}$.

Proof. As the Lie commutator $[\cdot, \cdot]$ is closed for subalgebras, (i) follows. Assuming that $\mathfrak{g}$ is simple we obtain (ii) as $\mathfrak{g}$ has no proper ideals and the adjoint representation has to be simple. The fact that the alternating square of the standard representation is isomorphic to the adjoint representation for $\mathfrak{s o}(k)$ with $k>1$ can be found on p. 353 of Ref. 1 or pages 199 and 213 of Ref. 17. The statement for $\mathfrak{s p}(\ell)$ is given on p. 353 of Ref. 1 and in Chap. VIII, Sec. 13, Ex. 8 of Ref. 17. Most of the corresponding highest weights are (e.g.,) given in Table 28 of Ref. 1, and the remaining ones statements can be directly verified.

\section{Appendix C: Case-by-case analysis for Alternating and Symmetric Squares}

In this appendix, we build on Appendix $B$ and provide a streamlined version of the laborious details needed for the proofs of Theorems 1 and 3.

\section{Alternating Squares}

In order to limit the case-by-case discussion, we reproduce a theorem and proof of Dynkin (see Thm. 4.5 of Ref. 1 ) which completely characterizes the irreducibility of the alternating squares of self-dual representations.

Theorem 36 (Dynkin). Let $\phi$ denote a self-dual and faithful representation of a compact semisimple Lie algebra $\mathfrak{g}$. The representation $\mathrm{Alt}^{2} \phi$ is not simple, if the pair $\left(\mathfrak{g}, x_{\phi}\right)$ is not contained in the following list: (a) ( $\mathfrak{s u}(2)$, (1)), (b) $(\mathfrak{s u}(2),(2))$, (c) $(\mathfrak{s o}(2 \ell+1),(1,0, \ldots, 0))$ with $\ell \geq 2$, and $(d)(\mathfrak{s o}(2 \ell),(1,0, \ldots, 0))$ with $\ell \geq 3$.

Proof. Due to Theorem 27 of Appendix A we can restrict us to simple representations $\phi$ of compact simple Lie algebras $\mathfrak{g}$. As $\phi$ is self-dual, we get either $(\mathrm{i}) \phi(\mathfrak{g}) \subseteq \mathfrak{s p}(\operatorname{dim}(\phi) / 2)$ or $(\mathrm{ii}) \phi(\mathfrak{g}) \subseteq \mathfrak{s o}(\operatorname{dim}(\phi))$. We consider first the case (i): Given the standard representation $\psi$ of $\mathfrak{s p}(\ell)$ with $x_{\psi}=(1,0, \ldots, 0)$ and $\ell>1$, we obtain that Alt ${ }^{2} \psi=\tau_{2} \oplus \tau_{0}$ decomposes into representations with highest weights $x_{\tau_{2}}=(0,1,0, \ldots, 0)$ and $x_{\tau_{0}}=(0, \ldots, 0)$ (see p. 360 in Ref. 1 , pp. 260-261 of Ref. 16, or pp. 206-207 of Ref. 17). It follows from Lemma 23 of Appendix A that Alt ${ }^{2} \phi$ cannot be simple for $\operatorname{dim}(\phi) \neq 2$. For $\operatorname{dim}(\phi)=2$, we get the element (a) from the list. Let us now treat the case (ii): In order to apply Lemma 23 of Appendix $\mathrm{A}$ again, we analyze the standard representation $\psi$ where $x_{\psi}=(1,0, \ldots, 0)$ for the Lie algebras $\mathfrak{s o}(k)$ with $k \geq 5, x_{\psi}=(2)$ for $\mathfrak{s o}(3) \cong \mathfrak{s u}(2)$, and $x_{\psi}=(1,1)$ for $\mathfrak{s o}(4) \cong \mathfrak{s u}(2) \oplus \mathfrak{s u}(2)$. Using Lemma 35 of Appendix B] one obtains that $\mathrm{Alt}^{2} \psi$ is isomorphic to the adjoint representation of the corresponding Lie algebras and that the adjoint representation is no longer simple when restricted to a proper subalgebra. Therefore, either $\mathfrak{g}=\mathfrak{s o}(2 \ell+1)$ or $\mathfrak{g}=\mathfrak{s o}(2 \ell)$ and we obtain the remaining elements in the list, while $\mathfrak{s o}(4) \cong \mathfrak{s u}(2) \oplus \mathfrak{s u}(2)$ is not $\operatorname{simple}$ and does not lead to a simple alternating square.

Consequently, we can limit us now to representations which are not self-dual. Due to Proposition 22 of Appendix A, we get that either $\mathfrak{g}=\mathfrak{s u}(\ell+1)$ with $\ell>1$, $\mathfrak{g}=\mathfrak{s o}(4 k+2)$, or $\mathfrak{g}=\mathfrak{e}_{6}$. The following lemma simplifies our search significantly.

Lemma 37. Let $\mathfrak{g}$ denote a compact simple Lie algebra which is simply-laced, i.e., $\mathfrak{s u}(\ell+1), \mathfrak{s o}(2 \ell), \mathfrak{e}_{6}, \mathfrak{e}_{7}$, or $\mathfrak{e}_{8}$. We consider the simple representations $\phi, \tau$, and $\psi$ where $\tau$ and $\psi$ are representations of $\mathfrak{g}$ and $\phi$ is a representation of a subalgebra $\mathfrak{h} \subseteq \mathfrak{g}$. We assume that $\phi \subseteq \tau \subseteq \psi$, i.e., that $\tau$ is subordinate to $\psi$ and that $\phi$ is a part of $\tau$. The representation $\mathrm{Alt}^{2} \psi$ is not simple if one of the following conditions holds:

(i) $x_{\phi}=(1,1)$ and either $\mathfrak{h}=\mathfrak{s u}(3)$ or $\mathfrak{h}=\mathfrak{s u}(2) \oplus \mathfrak{s u}(2)$.

(ii) More than one component $x_{\psi, j}$ of the highest weight $x_{\psi}$ is non-zero.

(iii) $x_{\phi}=(3)$ and $\mathfrak{h}=\mathfrak{s u}(2)$.

(iv) The highest weight $x_{\psi}$ has a component which is larger than two.

(v) $x_{\phi}=(0,2,0)$ and $\mathfrak{h}=\mathfrak{s u}(4)$.

(vi) The highest weight $x_{\psi}$ has a component which is larger than one and lies next to any end of the Dynkin diagram. (vii) $x_{\phi}=(0,0,1,0,0)$ and $\mathfrak{h}=\mathfrak{s u}(6)$.

(viii) The highest weight $x_{\psi}$ has a non-zero component which is not at or next to any end of the Dynkin diagram.

Proof. The non-irreducibility of $\mathrm{Alt}^{2} \phi$ for the cases in the statements (i), (iii), (v), and (vii) can be verified by direct computations. Then, all other statements follow via Propositions 32 and 34 of Appendix B 
TABLE V. Irreducible representations which are not self-dual and whose respective alternating square is irreducible (Dynkin)

\begin{tabular}{lllllll}
\hline \hline case & $\mathfrak{g}$ & $\ell$ & $\phi$ & $\operatorname{dim}(\phi)$ & $\operatorname{Alt}^{2} \phi$ & $(0,1,0, \ldots, 0)$ \\
\hline (i) & $\mathfrak{s u}(\ell+1)$ & $\ell \geq 2$ & $(1,0, \ldots, 0)$ & $\ell+1$ & $(2,1,0, \ldots, 0)$ & $\frac{\ell(\ell+1)}{2}$ \\
(ii) & $\mathfrak{s u}(\ell+1)$ & $\ell \geq 2$ & $(2,0, \ldots, 0)$ & $\frac{(\ell+1)(\ell+2)}{2}$ & $(1,0,1,0, \ldots, 0)$ & $3\left(\begin{array}{l}\ell+3 \\
4\end{array}\right)$ \\
(iii) & $\mathfrak{s u}(\ell+1)$ & $\ell \geq 3$ & $(0,1,0, \ldots, 0)$ & $\frac{\ell(\ell+1)}{2}$ & 16 & $(0,0,1,0,0)$ \\
(iv) & $\mathfrak{s o}(10)$ & - & $(0,0,0,1,0)$ & $(0,0,1,0,0,0)$ & 351 \\
(v) & $\mathfrak{e}_{6}$ & - & $(1,0,0,0,0,0)$ & 27 & & 120 \\
\hline \hline
\end{tabular}

Using Lemma 37 we can completely treat the case of simple representations which are not self-dual.

Lemma 38. Let $\phi$ denote a faithful representation of a compact semisimple Lie algebra $\mathfrak{g}$ such that $\phi$ is not self-dual and that the alternating square $\mathrm{Alt}^{2} \phi$ is simple. All possible cases (up to outer automorphisms of $\mathfrak{g}$ ) are given in Table $\mathrm{V}$.

Proof. Due to Theorem 27 of Appendix $\$$, $\phi$ is simple and $\mathfrak{g}$ is simple. Proposition 22 of Appendix $\mathrm{A}$ limits the possible cases to (a) $\mathfrak{g}=\mathfrak{s u}(\ell+1)$ with $\ell>1$, (b) $\mathfrak{g}=\mathfrak{s o}(4 k+2)$ with $k \geq 1$, or (c) $\mathfrak{g}=\mathfrak{e}_{6}$. Due to Lemma 37 only the cases (i)-(iii) of Table V can appear for (a). The irreducibility of the alternating square in the case (i) is well known (see p. 225 of Ref. 16 or p. 192 of Ref. 17). The case (ii)-(iii) are discussed (e.g.,) in Ex. 15.33 and 15.32 on pp. 226-227 of Ref. 16. This completes (a). For (b), one can directly verify that Alt ${ }^{2} \phi_{(0,0,0,0,0,1,0)}$ is not simple if $\mathfrak{g}=\mathfrak{s o}(14)$. We apply Propositions 32 and 34 of Appendix B and can now limit us to $\mathfrak{s o}(10)$, while $\mathfrak{s o}(6) \cong \mathfrak{s u}(4)$ has been already discussed. It can be directly checked that only the case (iv) of Table $\mathrm{V}$ remains. The Lie algebra $\mathfrak{g}=\mathfrak{e}_{6}$ from case (c) can also treated by explicit computations leading only to case (v) of Table V, which completes the proof.

The case-by-case analysis of Theorem 36 and Lemma 38 imply Theorem 1.

\section{Symmetric Squares}

In order to limit the case-by-case discussion, we reproduce a theorem of Dynkin (see Thm. 4.5 of Ref. 1) which completely treats the case of self-dual representations.

Theorem 39 (Dynkin). Let $\phi$ denote a self-dual and faithful representation of a compact semisimple Lie algebra $\mathfrak{g}$. The representation $\mathrm{Sym}^{2} \phi$ is not simple, if the pair $\left(\mathfrak{g}, x_{\phi}\right)$ is not equal to $(\mathfrak{s p}(\ell),(1,0, \ldots, 0))$.

Proof. Due to Theorem 27, we can limit us to simple representations $\phi$ of compact simple Lie algebras. As $\phi$ is selfdual, we get either (i) $\phi(\mathfrak{g}) \subseteq \mathfrak{s o}(\operatorname{dim}(\phi))$ or $($ ii $) \phi(\mathfrak{g}) \subseteq \mathfrak{s p}(\operatorname{dim}(\phi) / 2)$. We consider first the case (i): The Lie algebras $\mathfrak{s o}(\operatorname{dim}(\phi))$ with $\operatorname{dim}(\phi) \in\{1,2\}$ do not have simple subalgebras. The Lie algebra $\mathfrak{s o}(3) \cong \mathfrak{s u}(2) \cong \mathfrak{s p}(1)$ has no proper simple subalgebra, but leads to the case with $\mathfrak{g}=\mathfrak{s p}(1)$ and $x_{\phi}=(1)$. The symmetric $\operatorname{square~}^{2} \operatorname{Sym}_{(1,1)}=\psi_{(2,2)} \oplus \psi_{(0,0)}$ for the standard representation $\psi_{(1,1)}$ of $\mathfrak{s o}(4) \cong \mathfrak{s u}(2) \oplus \mathfrak{s u}(2)$ splits up into two components. So, $\left.\left(\right.$ Sym $\left.{ }^{2} \psi_{(1,1)}\right)\right|_{\mathfrak{g}}$ is also not simple. The symmetric square $\operatorname{Sym}^{2} \psi_{(1,0, \ldots, 0)}=\psi_{(2,0, \ldots, 0)} \oplus \psi_{(0, \ldots, 0)}$ for the standard representation $\psi_{(1,0, \ldots, 0)}$ of $\mathfrak{s o}(\operatorname{dim}(\phi))$ with $\operatorname{dim}(\phi) \geq 5$ decomposes (see Ex. 19.21 of Ref. 16), and no further cases can appear for (i). We analyze the case (ii): The standard representation $\psi:=\psi_{(1,0, \ldots, 0)}$ of $\mathfrak{s p}(\ell)$ has the symmetric square $\operatorname{Sym}^{2} \psi=\psi(2,0, \ldots, 0)$ which is simple and isomorphic to its adjoint representation and $\mathrm{Sym}^{2} \psi$ decomposes when restricted to a proper subalgebra (see Lemma 35 of Appendix B). It follows that $\mathfrak{g}=\mathfrak{s p}(\ell)$ which completes the proof.

Consequently, we can limit us again to representations which are not self-dual. Due to Proposition 22 of Appendix $\mathrm{A}$. we have either $\mathfrak{g}=\mathfrak{s u}(\ell+1)$ with $\ell>1, \mathfrak{g}=\mathfrak{s o}(4 k+2)$, or $\mathfrak{g}=\mathfrak{e}_{6}$. As in Section C1, we can simplify our analysis.

Lemma 40. Let $\mathfrak{g}$ denote a compact simple Lie algebra which is simply-laced, i.e., $\mathfrak{s u}(\ell+1), \mathfrak{s o}(2 \ell), \mathfrak{e}_{6}, \mathfrak{e}_{7}$, or $\mathfrak{e}_{8}$. We consider the simple representations $\phi, \tau$, and $\psi$ where $\tau$ and $\psi$ are representations of $\mathfrak{g}$ and $\phi$ is a representation of a subalgebra $\mathfrak{h} \subseteq \mathfrak{g}$. We assume that $\phi \subseteq \tau \subseteq \psi$, i.e., that $\tau$ is subordinate to $\psi$ and that $\phi$ is a part of $\tau$. The representation $\operatorname{Sym}^{2} \psi$ is not simple if one of the following conditions holds:

(i) $x_{\phi}=(1,1)$ and either $\mathfrak{h}=\mathfrak{s u}(3)$ or $\mathfrak{h}=\mathfrak{s u}(2) \oplus \mathfrak{s u}(2)$.

(ii) More than one component $x_{\psi, j}$ of the highest weight $x_{\psi}$ is non-zero. 
TABLE VI. Irreducible representations which are not self-dual and whose respective symmetric square is irreducible

\begin{tabular}{lllllll}
\hline \hline case & $\mathfrak{g}$ & $\ell$ & $\phi$ & $\operatorname{dim}(\phi)$ & $\operatorname{Sym}^{2} \phi$ & $\left.\operatorname{dim}^{2} \operatorname{Sym}^{2} \phi\right)$ \\
\hline$(\mathrm{i})$ & $\mathfrak{s u}(\ell+1)$ & $\ell \geq 2$ & $(1,0, \ldots, 0)$ & $\ell+1$ & $(2,0, \ldots, 0)$ & $\frac{(\ell+1)(\ell+2)}{2}$ \\
\hline \hline
\end{tabular}

(iii) $x_{\phi}=(2)$ and $\mathfrak{h}=\mathfrak{s u}(2)$.

(iv) The highest weight $x_{\psi}$ has a component which is larger than one.

(v) $x_{\phi}=(0,1,0)$ and $\mathfrak{h}=\mathfrak{s u}(4)$.

(vi) The highest weight $x_{\psi}$ has a non-zero component which is not at any end of the Dynkin diagram.

Proof. The non-irreducibility of $\operatorname{Sym}^{2} \phi$ for the cases in the statements (i), (iii), and (v) can be verified by direct computations. Then, all other statements follow via Propositions 32 and 34 of Appendix B.

Using Lemma 40 we can completely treat the case of simple representations which are not self-dual.

Lemma 41. Let $\phi$ denote a faithful representation of a compact semisimple Lie algebra $\mathfrak{g}$ such that $\phi$ is not self-dual and that the symmetric square $\mathrm{Sym}^{2} \phi$ is simple. All possible cases (up to outer automorphisms of $\mathfrak{g}$ ) are given in Table VI.

Proof. Due to Theorem 27 of Appendix $\mathrm{A}, \phi$ is simple and $\mathfrak{g}$ is simple. Proposition 22 of Appendix $\mathrm{A}$ limits the possible cases to (a) $\mathfrak{g}=\mathfrak{s u}(\ell+1)$ with $\ell>1$, (b) $\mathfrak{g}=\mathfrak{s o}(4 k+2)$ with $k \geq 1$, or (c) $\mathfrak{g}=\mathfrak{e}_{6}$. Due to Lemma 40 only the case (i) of Table VI can appear for (a). The irreducibility of the symmetric square in the case (i) is well known (see p. 225 of Ref. 16). For (b), one can directly verify that $\operatorname{Sym}^{2} \phi_{(0,0,0,1,0)}$ is not simple for $\mathfrak{g}=\mathfrak{s o}(10)$. We apply Propositions 32 and 34 of Appendix B and the remaining case of $\mathfrak{s o}(6) \cong \mathfrak{s u}(4)$ has been already discussed. One can check by explicit computation that $\phi_{(1,0,0,0,0,0)}, \phi_{(0,1,0,0,0,0)}$, and $\phi_{(0,0,0,0,0,1)}$ do not lead to a simple symmetric square for $\mathfrak{e}_{6}$ from (c), completing the proof.

The case-by-case analysis of Theorem 39 and Lemma 41 imply Theorem 3

\section{Appendix D: Generalizing a Theorem of Coquereaux and Zuber}

We provide here a straight-forward generalization of a result of Coquereaux and Zuber (see Proposition 43 below and Ref. 14) from the case of two simple representations of a simple, compact Lie algebra to the case of two semisimple representations of a compact Lie algebra. Recall that $\psi_{1} \otimes \psi_{2}$ denotes the outer tensor product as defined in Section A2. We start by observing a trivial property of the notation $\|\phi\|_{1}:=\sum_{i \in \mathcal{I}} m_{i}$.

Proposition 42. $\left\|\psi_{1} \otimes \psi_{2}\right\|_{1}=\left\|\psi_{1}\right\|_{1}\left\|\psi_{2}\right\|_{1}$ holds for two semisimple representations $\psi_{1}$ and $\psi_{2}$ of the compact Lie algebras $\mathfrak{g}_{1}$ and $\mathfrak{g}_{2}$, respectively.

A recent result of Coquereaux and Zuber connects the values of $\|\phi \otimes \psi\|_{1}$ and $\|\phi \otimes \bar{\psi}\|_{1}$.

Proposition 43 (Thm. 1 of Ref. 14). Given a compact simple Lie algebra $\mathfrak{g}$ and two simple representations $\phi$ and $\psi$ of $\mathfrak{g}$, it follows that $\|\phi \otimes \psi\|_{1}=\|\phi \otimes \bar{\psi}\|_{1}$.

Moreover, this is also valid for compact abelian Lie algebras as all their simple representations are one-dimensional.

Proposition 44. Let $\phi$ and $\psi$ denote simple representations of a compact abelian Lie algebra $\mathfrak{g}$, then $\|\phi \otimes \psi\|_{1}=$ $\|\phi \otimes \bar{\psi}\|_{1}=1$.

One can now generalize the theorem of Coquereaux and Zuber to simple representations of compact Lie algebras.

Proposition 45. Consider two simple representations $\phi$ and $\psi$ of a compact Lie algebra $\mathfrak{g}=\oplus_{\ell} \mathfrak{g}_{\ell}$ which can be decomposed into its simple and abelian subalgebras $\mathfrak{g}_{\ell}$. One obtains $\phi \cong \phi_{1} \otimes \phi_{2} \otimes \cdots \otimes \phi_{n}$ and $\psi \cong \psi_{1} \otimes \psi_{2} \otimes \cdots \otimes \psi_{n}$ for simple representations $\phi_{\ell}$ and $\psi_{\ell}$ of $\mathfrak{g}_{\ell}$. It follows that $\|\phi \otimes \psi\|_{1}=\|\phi \otimes \bar{\psi}\|_{1}$.

Proof. We note that $\phi \otimes \psi=\left(\otimes_{\ell} \phi_{\ell}\right) \otimes\left(\otimes_{\ell} \psi_{\ell}\right)=\otimes_{\ell}\left(\phi_{\ell} \otimes \psi_{\ell}\right)$ and $\phi \otimes \bar{\psi}=\left(\otimes_{\ell} \phi_{\ell}\right) \otimes\left(\otimes_{\ell} \bar{\psi}_{\ell}\right)=\otimes_{\ell}\left(\phi_{\ell} \otimes \bar{\psi}_{\ell}\right)$. One concludes from Proposition 42 that $\|\phi \otimes \psi\|_{1}=\prod_{\ell}\left\|\phi_{\ell} \otimes \psi_{\ell}\right\|_{1}$ and $\|\phi \otimes \bar{\psi}\|_{1}=\prod_{\ell}\left\|\phi_{\ell} \otimes \bar{\psi}_{\ell}\right\|_{1}$ hold. Applying Propositions 43 and 44. we get $\left\|\phi_{\ell} \otimes \psi_{\ell}\right\|_{1}=\left\|\phi_{\ell} \otimes \bar{\psi}_{\ell}\right\|_{1}$ for each $\ell$. Therefore, we obtain $\|\phi \otimes \psi\|_{1}=\|\phi \otimes \bar{\psi}\|_{1}$. 
All the previous propositions justify the following generalization of Proposition 43.

Proposition 46. Given a compact Lie algebra $\mathfrak{g}$ and two semisimple representations $\phi$ and $\psi$ of $\mathfrak{g}$, it follows that $\|\phi \otimes \psi\|_{1}=\|\phi \otimes \bar{\psi}\|_{1}$.

Proof. Consider the decompositions $\phi=\oplus_{\ell} \nu_{\ell}$ and $\psi=\oplus_{\kappa} \mu_{\kappa}$ of the representations $\phi$ and $\psi$ into its simple components, where the different components $\nu_{\ell}$ and $\mu_{\kappa}$ are not necessarily distinct. Proposition 7 (i) implies $\|\phi \otimes \psi\|_{1}=\| \oplus_{\ell, k}\left(\nu_{\ell} \otimes\right.$ $\left.\mu_{k}\right)\left\|_{1}=\sum_{\ell, k}\right\| \nu_{\ell} \otimes \mu_{k} \|_{1}$ and $\|\phi \otimes \bar{\psi}\|_{1}=\left\|\oplus_{\ell, k}\left(\nu_{\ell} \otimes \bar{\mu}_{k}\right)\right\|_{1}=\sum_{\ell, k}\left\|\nu_{\ell} \otimes \bar{\mu}_{k}\right\|_{1}$. We apply Proposition 45 and obtain $\left\|\nu_{\ell} \otimes \mu_{k}\right\|_{1}=\left\|\nu_{\ell} \otimes \bar{\mu}_{k}\right\|_{1}$. Hence, $\|\phi \otimes \psi\|_{1}=\|\phi \otimes \bar{\psi}\|_{1}$ also follows.

\section{Appendix E: Details on the semisimple part}

The proof of Theorem 16 will be stated in this appendix after presenting two auxiliary results which follow from Proposition 8 . Recall that a compact Lie algebra $\mathfrak{g}$ decomposes as $\mathfrak{g}=\mathfrak{s}(\mathfrak{g}) \oplus \mathfrak{c}(\mathfrak{g})$ where $\mathfrak{s}(\mathfrak{g})$ and $\mathfrak{c}(\mathfrak{g})$ denote its semsimple part and its center, respectively.

Corollary 47. Consider a subalgebra $\mathfrak{h}$ of a compact Lie algebra $\mathfrak{g}$ which observes $\mathfrak{h} \cap \mathfrak{c}(\mathfrak{g})=\mathfrak{c}(\mathfrak{g})$, i.e., one has the decompositions $\mathfrak{h}=[\mathfrak{h} \cap \mathfrak{s}(\mathfrak{g})] \oplus \mathfrak{c}(\mathfrak{g})$ and $\mathfrak{g}=\mathfrak{s}(\mathfrak{g}) \oplus \mathfrak{c}(\mathfrak{g})$. Note that $\mathfrak{h} \cap \mathfrak{s}(\mathfrak{g})$ might not be semisimple. A semisimple representation $\phi$ of $\mathfrak{g}$ decomposes as $\phi \cong \oplus_{i \in \mathcal{I}, k \in \mathcal{K}}\left[s_{i} \otimes c_{k}\right]^{\oplus m_{i k}}$ where $s_{i}$ (resp. $c_{k}$ ) denotes a simple representation of $\mathfrak{s}(\mathfrak{g})$ (resp. $\mathfrak{c}(\mathfrak{g})$ ). The corresponding multiplicity of the simple representation $s_{i} \otimes c_{k}$ is given by $m_{i k}$, and the classes of representations are indexed by $\mathcal{I}$ (resp. $\mathcal{K})$. Using the conditions

$$
\begin{aligned}
& \left\|\left.s_{i}\right|_{\mathfrak{h} \cap \mathfrak{s}(\mathfrak{g})}\right\|_{1}=\left\|s_{i}\right\|_{1}=1 \text { holds for all } i \in \mathcal{I} \text { with } m_{i k} \neq 0 \text { for some } k \in \mathcal{K}, \\
& \begin{aligned}
\left.\left(s_{i}\right)\right|_{\mathfrak{h} \cap \mathfrak{s}(\mathfrak{g})} \neq\left.\left(s_{\ell}\right)\right|_{\mathfrak{h} \cap \mathfrak{s}(\mathfrak{g})} & \text { holds for all } i, \ell \in \mathcal{I} \\
& \text { with } i \neq \ell, m_{i k} \neq 0, \text { and } m_{\ell k} \neq 0 \text { for some } k \in \mathcal{K},
\end{aligned}
\end{aligned}
$$

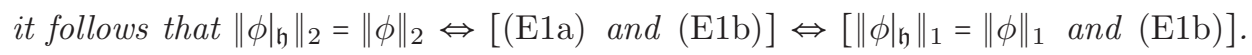

Proof. The restriction of $\mathfrak{g}$ to $\mathfrak{h}$ is trivial for the simple representations $c_{k}$ as $\mathfrak{h} \cap \mathfrak{c}(\mathfrak{g})=\mathfrak{c}(\mathfrak{g})$. Therefore, the corollary follows from Proposition 8 (c).

We apply now Corollary 47 to (not necessarily simple) representations and obtain the following useful lemma.

Lemma 48. We retain the notation of Corollary 47 and consider a decomposition $\phi \cong \oplus_{r \in R, k \in \mathcal{K}}\left[r \otimes c_{k}\right]^{\oplus m_{r k}}$ where $R$ denotes a suitably chosen set of (not necessarily simple) representations of $\mathfrak{h} \cap \mathfrak{s}(\mathfrak{g})$ and $m_{r k} \in\{0,1,2, \ldots\}$. It follows that the condition $\left\|\left.\phi\right|_{\mathfrak{h}}\right\|_{2}=\|\phi\|_{2}$ implies that $\left\|\left.r\right|_{\mathfrak{h} \cap \mathfrak{s}(\mathfrak{g})}\right\|_{2}=\|r\|_{2}$ holds for all $r \in R$ with $m_{r k} \neq 0$ for some $k \in \mathcal{K}$.

Proof. The condition $\left\|\left.\phi\right|_{\mathfrak{h}}\right\|_{2}=\|\phi\|_{2}$ implies via Corollary 47 that (E1a) and (E1b) hold for suitable representations $s_{i}$. But the same $s_{i}$ appear also in the decomposition of the representations $r$, and we apply Proposition 8 (c) to the subalgebra $\mathfrak{h} \cap \mathfrak{s}(\mathfrak{g})$ of $\mathfrak{s}(\mathfrak{g})$ to show that $\left\|\left.r\right|_{\mathfrak{h} \cap \mathfrak{s}(\mathfrak{g})}\right\|_{2}=\|r\|_{2}$.

After these preparations, we can provide the details for the proof of Theorem 16 .

Proof of Theorem 16. We have to show that $\mathfrak{s}(\mathfrak{h})=\mathfrak{s}(\mathfrak{g})$ is implied by $\operatorname{dim}\left(\mathfrak{c o m}\left[\left.(\phi \otimes \phi)\right|_{\mathfrak{h}}\right]\right)=\operatorname{dim}(\mathfrak{c o m}[\phi \otimes \phi])$. But as

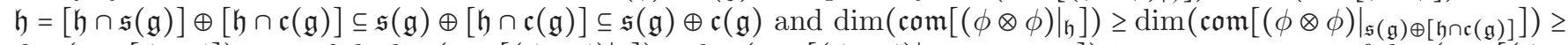

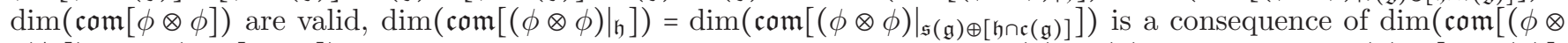
$\left.\left.\phi)\left.\right|_{\mathfrak{h}}\right]\right)=\operatorname{dim}(\mathfrak{c o m}[\phi \otimes \phi])$. Thus, the proof specializes to the case when $\mathfrak{h} \cap \mathfrak{c}(\mathfrak{g})=\mathfrak{c}(\mathfrak{g})$ holds, i.e., $\mathfrak{g}=\mathfrak{s}(\mathfrak{g}) \oplus[\mathfrak{h} \cap \mathfrak{c}(\mathfrak{g})]$ which we assume for the rest of the proof. Using the notation of Corollary 47 the representation $\phi$ decomposes as $\phi \cong \oplus_{i \in \mathcal{I}, k \in \mathcal{K}}\left[s_{i} \otimes c_{k}\right]^{\oplus m_{i k}}$. We apply the notation of Lemma 48 and obtain $\phi \otimes \phi \cong \oplus_{r \in R, k \in \mathcal{K}}\left[r \otimes c_{k}\right]^{\oplus m_{r k}}$ where $r \cong s_{i} \otimes s_{j}$ and $m_{r k} \in\{0,1,2, \ldots\}$. Lemma 48 implies that $\left\|\left.\left(s_{i} \otimes s_{i}\right)\right|_{\mathfrak{h} \cap \mathfrak{s}(\mathfrak{g})}\right\|_{2}=\left\|s_{i} \otimes s_{i}\right\|_{2}$ holds for each $i$. Recall that any ideal of a semsimple Lie algebra is a direct factor (cf. Chap. I, Sec. 6.4, Corollary to Prop. 5 of Ref. 20). As $\phi$ is faithful, one finds for every simple component $\mathfrak{g}_{\ell}$ of $\mathfrak{s}(\mathfrak{g}) \cong \oplus_{\ell} \mathfrak{g}_{\ell}$ an index $i$ such that $\operatorname{ker}\left(s_{i}\right) \cap \mathfrak{g}_{\ell}=\{0\}$ and $\operatorname{ker}\left(s_{i} \otimes s_{i}\right) \cap \mathfrak{g}_{\ell}=\{0\}$. Moreover, $\mathfrak{s}(\mathfrak{g}) / \operatorname{ker}\left(s_{i} \otimes s_{i}\right)$ is semisimple and we are able to apply Theorem 15 to the Lie algebra $\mathfrak{s}(\mathfrak{g}) / \operatorname{ker}\left(s_{i} \otimes s_{i}\right)$ and its faithful representation $s_{i} \otimes s_{i}$. This implies that $\mathfrak{h} \cap\left[\mathfrak{s}(\mathfrak{g}) / \operatorname{ker}\left(s_{i} \otimes s_{i}\right)\right]=\mathfrak{s}(\mathfrak{g}) / \operatorname{ker}\left(s_{i} \otimes s_{i}\right)$ for each $i$. In particular, we have that $\mathfrak{h} \cap \mathfrak{g}_{\ell}=\mathfrak{g}_{\ell}$ for each $\ell$ and the theorem follows. 


\section{Appendix F: Sharpening the bounds for the tensor-square theorem in the case of self-dual representations}

In this appendix, we prove Proposition 17 which under the assumption that $\mathfrak{g}$ is simple and $\alpha$ is self-dual provides bounds for the gap between the values of $\left\|\left.(\alpha \otimes \alpha)\right|_{\mathfrak{h}}\right\|_{1}$ and $\|\alpha \otimes \alpha\|_{1}$ (as well as $\left\|\left.(\alpha \otimes \alpha)\right|_{\mathfrak{h}}\right\|_{2}$ and $\left.\|\alpha \otimes \alpha\|_{2}\right)$ for any representation $\alpha$ of a compact semisimple Lie algebra $\mathfrak{g}$ and its restriction to a proper subalgebra $\mathfrak{h}$ of $\mathfrak{g}$. This generalizes inequalities given in Theorem[15. First, let us recall a result by King and Wybourne $\mathrm{19}^{\underline{9}}$.

Proposition 49 (Prop. 4.2 of 19 ). For any compact simple Lie algebra $\mathfrak{g}$, the multiplicity of occurrence of the adjoint representation $\theta$ in the tensor square of any self-dual and simple representation $\alpha$ is given by the number $b(\alpha)$ of non-vanishing components in the highest weight $\left(\alpha_{1}, \ldots, \alpha_{\ell}\right)$ corresponding to $\alpha$.

In order to apply Proposition 49, we derive bounds which depend on the multiplicity of the adjoint representation in the decomposition of $\phi \otimes \bar{\phi}$.

Lemma 50. Let $\phi$ denote a representation of the compact semisimple Lie algebra $\mathfrak{g}$, and let $\mathfrak{h}$ denote a proper subalgebra of $\mathfrak{g}$. If the multiplicity of the adjoint representation $\theta$ of $\mathfrak{g}$ in $\phi \otimes \bar{\phi}$ is $m$, then

(1) $\left\|\left.(\phi \otimes \bar{\phi})\right|_{\mathfrak{h}}\right\|_{1} \geq m+\|\phi \otimes \bar{\phi}\|_{1}$,

(2) $\left\|\left.(\phi \otimes \phi)\right|_{\mathfrak{h}}\right\|_{1} \geq m+\|\phi \otimes \phi\|_{1}$,

(3) $\left\|\left.(\phi \otimes \bar{\phi})\right|_{\mathfrak{h}}\right\|_{2} \geq m^{2}+\|\phi \otimes \bar{\phi}\|_{2}$,

(4) $\left\|\left.(\phi \otimes \phi)\right|_{\mathfrak{h}}\right\|_{2} \geq m^{2}+\|\phi \otimes \phi\|_{2}$,

(5) $\operatorname{dim}\left(\operatorname{com}\left[\left.(\phi \otimes \bar{\phi})\right|_{\mathfrak{h}}\right]\right) \geq m^{2}+\operatorname{dim}(\operatorname{com}[\phi \otimes \bar{\phi}])$,

(6) $\operatorname{dim}\left(\operatorname{com}\left[\left.(\phi \otimes \phi)\right|_{\mathfrak{h}}\right]\right) \geq m^{2}+\operatorname{dim}(\mathfrak{c o m}[\phi \otimes \phi])$.

Note that Proposition 17 follows now by combining Proposition 49 with Lemma 50,

Proof of Lemma 50. We decompose $\phi \otimes \bar{\phi}$ into $\theta^{\oplus m} \oplus \psi$ with maximal $m$. Proposition 12 implies that $\left\|\left.\theta\right|_{\mathfrak{h}}\right\|_{1} \geq\|\theta\|_{1}+1$. Applying this inequality and Proposition $7(\mathrm{i})$, we obtain $\left\|\left.(\phi \otimes \bar{\phi})\right|_{\mathfrak{h}}\right\|_{1}=\left\|\left.\theta^{\oplus m}\right|_{\mathfrak{h}}\right\|_{1}+\left\|\left.\psi\right|_{\mathfrak{h}}\right\|_{1}=m\left\|\left.\theta\right|_{\mathfrak{h}}\right\|_{1}+\left\|\left.\psi\right|_{\mathfrak{h}}\right\|_{1} \geq$ $m\left(\|\theta\|_{1}+1\right)+\|\psi\|_{1}=m+\left\|\theta^{\oplus m}\right\|_{1}+\|\psi\|_{1}=m+\|(\phi \otimes \bar{\phi})\|_{1}$, which proves (1). Statement (2) follows from (1) via Proposition [46. Consider the decompositions $\theta \cong \oplus_{i} \nu_{i}^{\oplus m_{i}}$ and $\psi \cong \oplus_{i} \nu_{i}^{\oplus n_{i}}$ into simple representations $\nu_{i}$. It follows that $\phi \otimes \bar{\phi}=\oplus_{i} \nu_{i}^{\oplus\left(m m_{i}+n_{i}\right)}$. Since $\left\|\left.\theta\right|_{\mathfrak{h}}\right\|_{1} \geq\|\theta\|_{1}+1$, we get from Proposition $8(\mathrm{a})$-(b) that $\left\|\left.\left(\nu_{p}\right)\right|_{\mathfrak{h}}\right\|_{2} \geq\left\|\nu_{p}\right\|_{2}+1$ holds for some $\nu_{p}$ with $m_{p} \geq 1$. Thus, one can write $\left\|\left.\phi\right|_{\mathfrak{h}}\right\|_{2}=\left\|\left.\oplus_{i}\left(\nu_{i}\right)\right|_{\mathfrak{h}} ^{\oplus\left(m m_{i}+n_{i}\right)}\right\|_{2}=\sum_{i}\left(m m_{i}+n_{i}\right)^{2}\left\|\left.\left(\nu_{i}\right)\right|_{\mathfrak{h}}\right\|_{2}=\left(m m_{p}+n_{p}\right)^{2}\left\|\left.\left(\nu_{p}\right)\right|_{\mathfrak{h}}\right\|_{2}+$ $\sum_{i \neq p}\left(m m_{i}+n_{i}\right)^{2}\left\|\left.\left(\nu_{i}\right)\right|_{\mathfrak{h}}\right\|_{2} \geq\left(m m_{p}+n_{p}\right)^{2}\left(\left\|\nu_{p}\right\|_{2}+1\right)+\sum_{i \neq p}\left(m m_{i}+n_{i}\right)^{2}\left\|\nu_{i}\right\|_{2}=\left(m m_{p}+n_{p}\right)^{2}+\|\phi \otimes \bar{\phi}\|_{2} \geq m^{2}+\|\phi \otimes \bar{\phi}\|_{2}$. This completes the proof of (3). Statement (4) follows via Proposition 10, Obviously, (5) and (6) are a consequence of (3) and (4), respectively.

${ }^{1}$ E. B. Dynkin, Amer. Math. Soc. Transl. Ser. 2 6, 245 (1957), reprinted in Ref. 3

${ }^{2}$ E. B. Dynkin, Amer. Math. Soc. Transl. Ser. 2 6, 111 (1957), reprinted in Ref. 3 .

${ }^{3}$ E. B. Dynkin, Selected Papers of E. B. Dynkin with Commentary (American Mathematical Society and International Press, 2000).

${ }^{4}$ R. Zeier and T. Schulte-Herbrüggen, J. Math. Phys. 52, 113510 (2011).

${ }^{5}$ D. Gross, K. Audenaert, and J. Eisert, J. Math. Phys. 48, 052104 (2007).

${ }^{6}$ A. Roy and A. J. Scott, Des. Codes Cryptogr. 53, 13 (2009).

${ }^{7}$ Z. Zimborás, in Proceedings of the 6th International Workshop on Lie Theory and its Applications in Physics, Varna, Bulgaria, 15-21 August 2005 (Heron Press, Sofia, 2006) pp. 76-82, see also arxiv.org/abs/math/0512107

${ }^{8}$ J. R. McMullen, Math. Z. 185, 539 (1984).

${ }^{9}$ D. Handelman, Int. J. Math. 4, 59 (1993).

${ }^{10}$ D. Kazhdan, M. Larsen, and Y. Varshavsky, Algebra \& Number Theory 8, 243 (2014).

${ }^{11}$ M. Larsen and R. Pink, Invent. math. 102, 377 (1990).

${ }^{12}$ M. Larsen, Int. Math. Res. Notices 2004, 1989 (2004).

${ }^{13}$ J. An, J.-K. Yu, and J. Yu, J. Diff. Geom. 94, 59 (2013).

${ }^{14}$ R. Coquereaux and J.-B. Zuber, J. Phys. A 44, 295208 (2011).

${ }^{15}$ Z. Zimborás, R. Zeier, M. Keyl, and T. Schulte-Herbrüggen, EPJ Quantum Technology 1, 11 (2014).

${ }^{16}$ W. Fulton and J. Harris, Representation Theory: A First Course (Springer, New York, 1991).

${ }^{17}$ N. Bourbaki, Elements of Mathematics, Lie Groups and Lie Algebras, Chapters 7-9 (Springer, Berlin, 2008).

${ }^{18}$ Z. Zimborás, R. Zeier, T. Schulte-Herbrüggen, and D. Burgarth, "Symmetry decides quantum simulability of effective interactions," (2015), arXiv:1504.07734 [quant-ph].

${ }^{19}$ R. C. King and B. G. Wybourne, J. Phys. A 29, 5059 (1996).

${ }^{20}$ N. Bourbaki, Elements of Mathematics, Lie Groups and Lie Algebras, Chapters 1-3 (Springer, Berlin, 1989).

${ }^{21}$ S. Helgason, Differential Geometry, Lie Groups, and Symmetric Spaces (Academic Press, New York, 1978).

${ }^{22} \mathrm{~W}$. Ledermann, Introduction to Group Characters, 2nd ed. (Cambridge University Press, Cambridge, 1987).

${ }^{23}$ H. Samelson, Notes on Lie Algebras, 2nd ed. (Springer, New York, 1990).

${ }^{24}$ N. Bourbaki, Elements of Mathematics, Lie Groups and Lie Algebras, Chapters 4-6 (Springer, Berlin, 2008).

${ }^{25}$ A. I. Malcev, Amer. Math. Soc. Transl. 33 (1950).

${ }^{26}$ A. K. Bose and J. Patera, J. Math. Phys. 11, 2231 (1970). 
${ }^{27}$ W. G. MacKay and J. Patera, Tables of Dimensions, Indices, and Branching Rules for Representations of Simple Lie Algebras (Marcel Dekker, New York, 1981).

${ }^{28}$ J. M. Landsberg, Tensors: Geometry and Applications (American Mathematical Society, Providence, 2012).

${ }^{29}$ J. A. Wolf, Acta Math. 120, 59 (1968).

${ }^{30}$ N. Bourbaki, Éléments de Mathématique, Algèbre, Chapitre 8, 2nd ed. (Springer, Berlin, 2012).

${ }^{31}$ M. Goto and F. D. Grosshans, Semisimple Lie Algebras (Marcel Dekker, New York, 1978).

${ }^{32}$ H. Aslaksen, J. Pure Appl. Algebra 93, 135 (1994).

${ }^{33}$ R. N. Cahn, Semi-Simple Lie Algebras and Their Representations (The Benjamin/Cummings Publishing Company, Menlo Park, 1984).

${ }^{34}$ M. Krämer, Reps. Math. Phys. 13, 295 (1978). 